\title{
Distributions of Galaxy Spectral Types in the Sloan Digital Sky Survey
}

\author{
C. W. Yip ${ }^{1}$, A. J. Connolly ${ }^{1}$, A. Szalay², T. Budavari², M. SubbaRao ${ }^{3}$, J. Frieman ${ }^{3}$, \\ R. Nichol ${ }^{4}$, A. Hopkins ${ }^{1}$, D. York ${ }^{3,5}$, S. Okamura 6 , J. Brinkmann ${ }^{7}$, I. Csabai ${ }^{2,8}$, \\ A. R. Thakar ${ }^{2}$, M. Fukugita ${ }^{9}$, Z. Ivezić ${ }^{10}$
}

\begin{abstract}
We perform an objective classification of 170,000 galaxy spectra in the Sloan Digital Sky Survey (SDSS) using the Karhunen-Loève (KL) transform. With about one-sixth of the total set of galaxy spectra which will be obtained by the survey, we are able to carry out the most extensive analysis of its kind to date. The formalism proposed by Connolly and Szalay (1999a) is adopted to correct for gappy regions in the spectra, and to derive eigenspectra and eigencoefficients. From this analysis, we show that this gap-correction formalism leads to a converging set of eigenspectra and KL-repaired spectra. Furthermore, KL eigenspectra of galaxies are found to be convergent not only as a function of iteration, but also as a function of the number of randomly selected galaxy spectra used in the analysis. From these data a set of ten eigenspectra of galaxy spectra are constructed, with rest-wavelength coverage $3450-8350 \AA$. The eigencoefficients describing these galaxies naturally place the spectra into several classes defined by the plane formed by the first three eigencoefficients of each spectrum. Spectral types, corresponding to different Hubble-types and galaxies with extreme emission lines, are identified for the 170,000 spectra and are shown to be complementary to existing spectral classifications. From a non-parametric classification technique, we find that the population of galaxies can be divided into three classes which correspond to early late- through to intermediate late-types galaxies. This finding is believed to be related to the color separation of SDSS galaxies discussed in earlier works. Bias in the spectral classifications due to the aperture spectroscopy in the SDSS is small and within the signal-to-noise limit for majority of galaxies except for the reddest nearby galaxies and large galaxies $(>30 \mathrm{kpc})$ with prominent emissions. The mean spectra and eigenspectra derived from this work can be downloaded from http://www.sdss.org.
\end{abstract}

Subject headings: galaxies:fundamental parameters - galaxies:general - methods: data analysis - techniques: spectroscopic

\footnotetext{
${ }^{1}$ Department of Physics and Astronomy, University of Pittsburgh, Pittsburgh, PA 15260, USA

${ }^{2}$ Department of Physics and Astronomy, Johns Hopkins University, 3701 San Martin's Drive, Baltimore, MD21218, USA

${ }^{3}$ Department of Astronomy and Astrophysics, The University of Chicago, 5640 S. Ellis Ave, Chicago, IL, 60637 USA

${ }^{4}$ Department of Physics, Carnegie Mellon University, 5000 Forbes Ave., Pittsburgh, PA 15213, USA

${ }^{5}$ Enrico Fermi Institute, The University of Chicago, 5640 South Ellis Avenue, Chicago, IL 60637, USA

${ }^{6}$ Department of Astronomy and Research Center for the
}

\footnotetext{
Early Universe, School of Science, University of Tokyo, Tokyo 113-0033, Japan

${ }^{7}$ Apache Point Observatory, 2001 Apache Point Road, P.O. Box 59, Sunspot, NM88349-0059, USA

${ }^{8}$ Department of Physics, Eötvös University, Budapest, Pf. 32, Hungary, H-1518

${ }^{9}$ Institute for Cosmic Ray Research, University of Tokyo, Japan

${ }^{10} 2$ Peyton Hall, Princeton University, Princeton, NJ 08544, USA
} 


\section{Introduction}

The most successful scheme used to date to classify galaxies is the morphological classification of Hubble (Hubble 1926). The utility of this simple classification scheme (a compression of the available morphological types to approximately seven classes) has become apparent through its application to numerous extragalactic studies. Spectral classifications have a number of natural advantages over the morphological classifications of Hubble in that they are more easily related to the physical processes that are ongoing within a galaxy (e.g., star formation) and that they do not require us to obtain high resolution imaging of a large number of galaxies. As such, they are well suited to studying the cores of galaxies in the distant universe.

As was found for the classification of the spectra of stars, classifying the spectra of more complicated systems such as galaxies or quasars (QSOs) can provide a better understanding of the physical processes that determine the formation and evolution of these sources. Moreover, if there exist mechanisms by which galaxies can be classified using a handful of representative parameters, this classification can be thought of as a compression of the information contained within the spectra. From such an approach, one might be able to derive simple mechanisms for exploring the physics of the spectral properties of galaxies using large data sets. Recent massive spectroscopic surveys, e.g., the Anglo-Australian Observatory 2-degree-Field (2dF) Galaxy Survey (Colless et al. 2001) and Sloan Digital Sky Survey (SDSS; York et al. 2000) provide us with the opportunity to address the classification of galaxy spectra using hundreds of thousands of galaxy spectral energy distributions (SEDs). One technique that has gained popularity for studying the distribution of SEDs is the Karhunen-Loève (KL) transform. The power of this approach is that it enables a large amount of data to be decomposed and compressed into independent components in an objective way. Applications of this technique can be found in the classifications of galaxies (Connolly et al. 1995; Folkes et al. 1996; Sodre \& Cuevas 1997; Bromley et al. 1998; Galaz \& de Lapparent 1998; Ronen et al. 1998; Folkes et al. 1999), QSOs (Francis et al. 1992;
Boroson \& Green 1992; Yip et al. 2004) and stars (Singh et al. 1998; Bailer-Jones et al. 1998).

This paper is organized as follows. In Section 2, we describe the spectral data used in our analysis. In Section 3, we discuss the details of the Karhunen-Loève transform and the gap-correction formalism. In Section 4, we pose the problems to be addressed with this paper, and show the results of a convergence analysis on the KL gap-correction formalism. In Section 5, we derive the eigenspectra and eigencoefficients for the full SDSS data set, and implement a classification scheme. In Section 6, we discuss the reliability of this classification. A simple model is used to describe the population of galaxies in Section 7. In Section 8, we discuss the applications of the KL eigenspectra obtained in this work. In Section 9, we discuss the aperture bias effect on the current classification scheme. Finally, in Section 10 we conclude our results and discuss some possible future directions based on this work.

\section{Data}

As part of the Sloan Digital Sky Survey (York et al. 2000) spectra are taken with fibers of 3 arcsec diameter (corresponding to $0.18 \mathrm{~mm}$ at the focal plane for the $2.5 \mathrm{~m}, \mathrm{f} / 5$ telescope). All sources are selected from an initial imaging survey using the SDSS camera described in Gunn et al. (1998) with the filter response curves as described in Fukugita et al. (1996), and using the imaging processing pipeline of Lupton et al. (2000). The astrometric calibration is described in Pier et al. (2002). The photometric system and monitoring are described in detail in Smith et al. (2002) and Hogg et al. (2001) respectively. To date, there are three complete samples of SDSS spectra: the Main Galaxy sample (Strauss et al. 2002), the Luminous Red Galaxy sample (LRGs; Eisenstein et al. 2002), and the QSO sample (Richards et al. 2002). From these data we select the Main Galaxy sample for our analysis and use only those galaxies defined as being of survey quality: a signal-tonoise lower-limit of approximately 16 . The galaxies in this sample have r-band Petrosian magnitudes $r_{p}<17.77$ and Petrosian half-light surface brightnesses $\mu_{50}<24.5 \mathrm{mag} \operatorname{arcsec}^{-2}$, defined to be the mean surface brightness within a circular aperture containing half of the Petrosian flux 
(called the Petrosian half-light radius). The spectral reductions used are the standard SDSS 2D analysis pipeline (idlspec2D v4.9.8, as of $18 \mathrm{th}$ of April, 2002) and the 1D SpecBS pipeline (Schlegel et al. 2003). The resultant spectra are flux- and wavelength-calibrated, and sky-subtracted. From these data approximately two hundred galaxies are removed, as they have zero flux in all pixels. This results in a final sample of 176,956 galaxy spectra. The median redshift is about 0.1 , and we find that about $6.5 \%$ of the sample have redshifts $c z<10,000 \mathrm{kms}^{-1}$, so that their Petrosian halflight radii can be substantially larger than the 3 arcsec aperture of the fiber (Strauss et al. 2002). 1,854 spectra of the final sample are found to be duplicated observations; identified as being within a search radius of 2 arcsec and a redshift tolerance of 0.01 . All spectra are shifted to a common rest frame, and rebinned to a vacuum wavelength coverage of $3450-8350 \AA$. The binning of the spectra is logarithmic, with a velocity dispersion of $69 \mathrm{kms}^{-1}$. This procedure emphasizes the blue end of the optical spectrum, enabling our analysis to focus on the $\mathrm{Ca} \mathrm{H}$ and $\mathrm{Ca} \mathrm{K}$ lines, and the Balmer break. The resultant spectra cover restwavelength range $3450-8350 \AA$ over 3839 pixels.

\section{KL and Gap-Correction Formalism}

The Karhunen-Loève transform (or Principal Component Analysis, PCA) is a powerful technique used in classification and dimensional reduction of data. In astronomy, its applications in studies of multivariate distributions have been discussed in detail (Efstathiou \& Fall 1984; Murtagh \& Heck 1987). In this paper, we limit ourselves to its applications to spectral energy distributions. The basic idea is to derive a lower dimensional set of eigenspectra (Connolly et al. 1995) from a very large set of input SEDs. Each SED can be thought of as an axis in a multidimensional hyperspace, $f_{\lambda_{k} i}$, where $\lambda_{k}$ denotes the $k$-th wavelength in the $i$-th galaxy spectrum.

For the moment, we assume that there are no gaps in each spectrum; we will discuss the ways we deal with gappy regions later. From the set of spectra we construct the correlation matrix

$$
C_{\lambda_{k} \lambda_{l}}=\hat{f}_{\lambda_{k} i} \hat{f}_{i \lambda_{l}}
$$

where the summation is from $i=1$ to the total number of spectra, and $\hat{f}_{\lambda_{k} i}$ is the normalized $i$-th spectrum, defined for a given $i$ as,

$$
\hat{f}_{\lambda_{k}}=\frac{f_{\lambda_{k}}}{\sqrt{f_{\lambda_{k}} \cdot f_{\lambda_{k}}}}
$$

The eigenspectra are obtained by finding a matrix, $U$, such that

$$
U^{T} C U=\Lambda
$$

where $\Lambda$ is the diagonal matrix containing the eigenvalues of the correlation matrix. $U$ is thus a matrix whose $i$-th column consists of the $i$-th eigenspectrum $e_{i \lambda_{k}}$. We solve this eigenvalue problem by using Singular Value Decomposition.

The observed spectra are projected on to the eigenspectra to obtain the eigencoefficients. In these projections, every pixel in each spectrum is weighted by the error associated with that particular pixel, $\sigma_{\lambda}$, such that the weights are given by $w_{\lambda}=1 / \sigma_{\lambda}{ }^{2}$. The observed spectra can be decomposed, with no error, as follows

$$
f_{\lambda_{k}}=\sum_{i=1}^{M} a_{i} e_{i \lambda_{k}}
$$

where $M$ is the total number of eigenspectra. It is straightforward to see that $M$ equals the total number of wavelength bins in the spectrum.

Previously, we assumed that the spectra are without any gaps. In reality, however, there are several reasons for gaps to exist: for example, different rest-wavelength coverages, the removal of sky lines, bad pixels on the CCD chips etc. leave gaps at different rest frame wavelengths for each spectrum. All can contribute to incomplete spectra. The principle behind the gap-correction process is to reconstruct the gappy spectrum using its principal components. The first application of the method to analyze galaxy spectra is due to Connolly and Szalay (1999a), which expands on a formalism developed by Everson and Sirovich for dealing with two-dimensional images (Everson \& Sirovich 1994). Initially, we fix the gap regions by some means, for example, linearinterpolation. A set of eigenspectra are then constructed from the gap-repaired galaxy spectra. Afterward, the gaps in the original spectra are corrected with the linear combination of the KL eigenspectra. The whole process is iterated until 
the eigenspectra converge, which we define in the next section. According to Everson and Sirovich's work on artificially masked two-dimensional images, they claimed that the iteration process gives convergent images. However, the question about whether the principal components resulting from the correction procedure on realistic gappy galaxy spectra would converge is unknown and is to be addressed with this work.

\section{A Convergence Analysis of KL}

There are some questions to be solved in our analysis before the KL eigenspectra and hence the classification itself become robust and meaningful. These questions are: do the resultant eigenspectra converge and, if so, how many iteration steps are required, what is the dependency of the quality of the KL-repaired spectra on how the gaps are initially corrected, how much information is contained in the eigenspectra and most importantly, how many galaxy spectra are needed in order to derive a convergent set of eigenspectra?

Several authors have tried to assess the performance of a KL analysis in a number of different quantitative ways. An example of this is the $\chi^{2}$ assessment (Francis et al. 1992) in which the authors calculated the difference between the observed spectrum and the spectrum reconstructed with the principal components in order to determine the number of components needed for reconstructing a quasar spectrum. With the implementation of gap-corrections in our analysis, this comparison of only one spectrum to another may not suffice. We are more interested in how well the set of eigenspectra describes the distribution of spectra rather than a one-to-one comparison. For example, how does the set of eigenspectra differ as the gap-correction procedure progresses? Given two subspaces, each formed by a set of eigenspectra obtained with different conditions (e.g., at different points in the iterative gap correction or computed with different numbers of observed spectra), we require a method that will quantitatively compare one set of eigenspectra with another. In other words, instead of just comparing two spectra, a mechanism is desired to compare two subspaces, which are spanned by a finite number of spectra respectively. Mathematically it can be stated, as in (Everson \& Sirovich 1994), that two spaces, $E$ and $F$, are in common if

$$
\operatorname{Tr}(\mathbf{E F E})=D,
$$

where $\mathbf{E}$ and $\mathbf{F}$ are the sum of projection operators of space $E$ and $F$ respectively, and $D$ is equal to the dimensionality of each space. We assume that these eigenbases have the same dimensions for a meaningful comparison. The sum of the projection operators, $\mathbf{E}$, of a space is given by the sum of the outer products

$$
\mathbf{E}=\sum_{\epsilon}|\epsilon><\epsilon|
$$

where $\mid \epsilon>$ are the basis vectors which span the space $E$ (e.g., Merzbacher 1970). A basis vector is an eigenspectrum if $E$ is considered to be a set of eigenspectra. The two spaces are disjoint if the trace quantity is zero and are identical if the quantity is equal to the dimension of the subspace. This provides a quantitative way of measuring the commonality of two subspaces (i.e., how similar the two subspaces are).
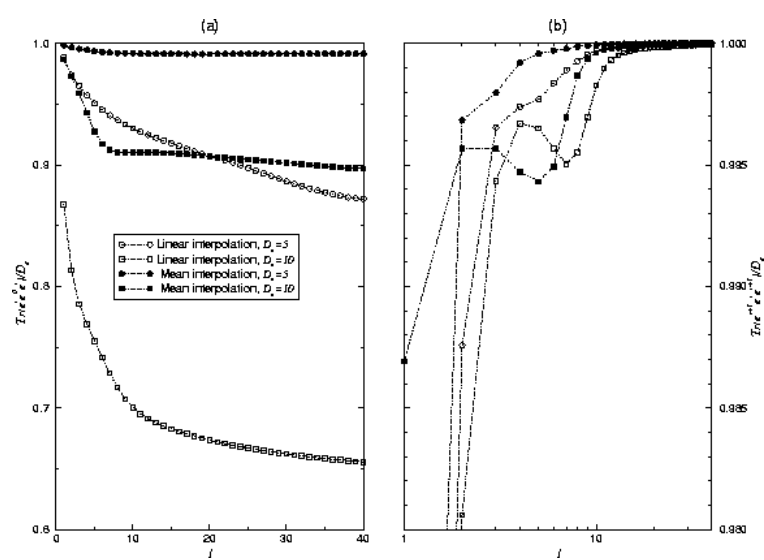

Fig. 1. - Convergence of eigenspectra (a) $\operatorname{Tr}\left(e^{i} e^{0} e^{i}\right)$ and (b) $\operatorname{Tr}\left(e^{i+1} e^{i} e^{i+1}\right)$ as a function of iteration step in the KL gap-correction formalism. Curves with open symbols are linearlyinterpolated across the gaps while filled symbols represent mean-interpolated data. The circles and squares denote $D_{e}=5$ and 10 respectively, where $D_{e}$ is the dimension of the subspace formed by the eigenspectra.

In investigating the convergence behavior of eigenspectra as a function of the number of iterations, we define one of the spaces to be that formed 
by a finite number of eigenspectra obtained after initially interpolating over the gap regions, but without gap correction. The other spaces are defined to be those formed by the same finite number of eigenspectra but at different iterations in the gap correction. The sum of projection operators in the first case is named $e^{0}$ and in the latter, $e^{i}$. The subspaces are named $E^{0}$ and $E^{i}$ respectively, where $i$ denotes the $i$-th iteration. The dimension of the space is $D_{e}$, which is the number of eigenspectra forming the subspace.

The trace quantity $\operatorname{Tr}\left(e^{i} e^{0} e^{i}\right)$ as a function of iteration is plotted in Figure 1a, in which the KL transform is applied to $N=4003$ randomlychosen SDSS galaxy spectra, where $D_{e}$ is set to be 5 and 10, meaning that the subspaces are spanned by the the first five and the first ten eigenspectra respectively. Repairing of the galaxy spectra in the iteration procedure is performed with $m=10$ eigenspectra. It should be noted that $m$ is independent from $D_{e}$ and that $D_{e}$ is always smaller or equal to $m$. The traces are normalized by the corresponding $D_{e}$ in each curve to simplify comparison. Initially, let us concentrate on the curves with open symbols in Figure 1. These curves denote that the initial gappy regions are approximated by linear-interpolation. In this linear-interpolation method the flux of a pixel, $f_{\lambda_{k}}^{g}$, in the gap is simply approximated by the average of its neighbors, so that

$$
f_{\lambda_{k}}^{g}=\left(f_{\lambda_{k-1}}+f_{\lambda_{k+1}}\right) / 2
$$

The trace quantity decreases gradually as the iteration step increases, indicating that the space $E^{i}$ is less and less in common with $E^{0}$. This implies that the KL gap-correction and eigenspectra construction are changing the spectrum of a galaxy within the gappy regions. As such the eigenspectra from the KL-repaired spectra differ progressively more from those formed from the original spectra. The above is true for both $D_{e}=5$ and 10. The above is generally valid for $D_{e}$ from $1-10$. As the iteration increases, the slope of the curve decreases, which implies that a we have converging set of eigenspectra.

The choice of linear-interpolation in the initial correction for the gappy regions is arbitrary. In fact, if the gap formalism is robust, the quality of the KL-repaired spectrum and the eigenspectra should be independent of the way the observed spectra are initially repaired. We test an alternative method of correcting for gaps, where the flux at each wavelength bin in the gap region is approximated by the mean of all other spectra within that region, i.e., the flux of a pixel in a gap is approximated by,

$$
\hat{f}_{\lambda_{k}}^{g}=\left\langle\sum_{\text {all spec }} \hat{f}_{\lambda_{k}}\right\rangle_{\text {all spec }}
$$

where $\hat{f}_{\lambda_{k}}$ is the normalized flux at $\lambda_{k}$. We call this method mean-interpolation. With this alternate method the trace quantity also converges as we can see from Figure 1a, but at a higher value than those in the case of linearinterpolation. This behavior shows that, in the case of mean-interpolation, the eigenspectra constructed after the gap correction deviate less from the initial interpolated spectra than in the case of linear-interpolation. The rate of convergence is faster when using the mean-interpolation method. This suggests that the mean-interpolation provides a better initial estimate of the true spectra within the gap regions. We do not, therefore, require as many iterations as in the case of linearinterpolation. This is important as each step in repairing the spectra and constructing the eigenspectra is computationally expensive when large amounts of data are under consideration.

Figure $1 \mathrm{~b}$ shows the convergence behavior of the sets of eigenspectra given in Figure 1a except that the trace quantity now compares the subspace from one iteration to the subspace from the next iteration, i.e., $\operatorname{Tr}\left(e^{i+1} e^{i} e^{i+1}\right)$. As expected, the convergence with the number of iteration steps can be seen in both methods, but with this more sensitive measurement the convergence is now no longer found to be monotonic. This implies that we may need more iterations, than it first appeared from our previous example in order to obtain a convergent set of eigenspectra. Again, the mean-interpolation method is shown to converge to a consistent set of eigenspectra faster than for linear-interpolation. Consequently, in the following all gaps in the spectra will be fixed using the mean-interpolation method, unless otherwise specified.

An example of the actual performance in the mean-interpolated and repaired spectra is shown in Figure 2. The data set is the same 4003 galax- 

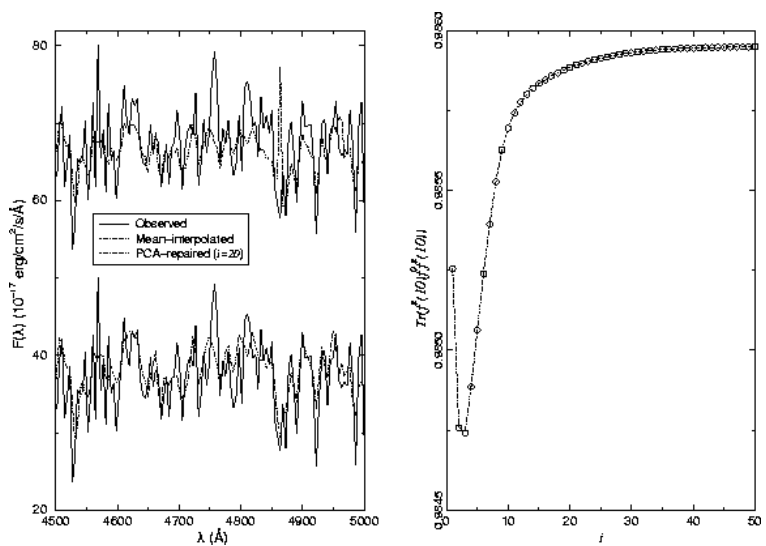

Fig. 2.- (a) The mean-interpolated and KLrepaired (20 iterations) of an artificially masked spectra, overlaid on the original unmasked spectrum. (b) KL-repaired spectra converge as a function of iteration.

ies as before, except that one randomly chosen spectrum is artificially masked in the region $[4500$, $5000]$ A . The upper panel of Figure 2a shows that the mean-interpolated region is already close to that of the original spectrum before masking. The lower panel shows the KL-repaired spectrum at $i=20$ overlaid on the original spectrum, using all 10 eigenspectra. The spectra are offset by an arbitrary amount for illustration. There is a substantial improvement in retrieving the original spectrum as the iterations proceed. To compare the KL-repaired and unmasked spectrum quantitatively, we apply a similar convergence measure as described previously. The convergence measure in this case is defined to be

$$
\operatorname{Tr}\left(f^{R}(m) f^{0} f^{R}(m)\right),
$$

where $f^{R}(m)$ is the projector of the KL-repaired spectrum with $m$ eigenspectra in the gaps, and $f^{0}$ is that of the unmasked spectrum. In Figure 2b, the trace quantity versus the number of iterations is plotted for the case corresponding to Figure 2a. $\operatorname{Tr}\left(f^{R}(m) f^{0} f^{R}(m)\right)=1$ means that the repaired spectrum is identical with the original unmasked one. We see that after the initial few iterations, the two become more similar. After 30 iterations, the KL-repaired spectrum converges to that of the original spectrum with a high degree of accuracy, the difference in the trace quantities of $8 \times 10^{-4} \%$.

Combining the results of the convergence mea-

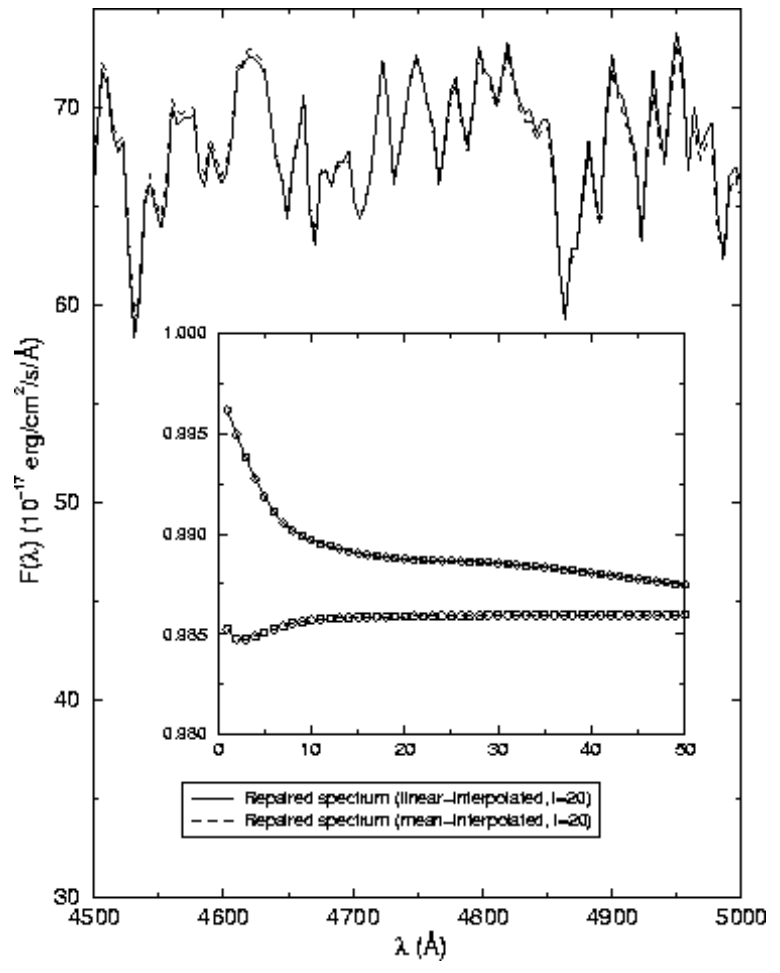

Fig. 3. - The KL-repaired spectra, linearinterpolated (solid line) and mean-interpolated (broken line), in the gap regions of the unmasked spectrum as in Figure 2 (after 20 iterations). The insert is $\operatorname{Tr}\left(f^{R}(m) f^{0} f^{R}(m)\right)$ as a function of iteration step for both cases.

sures in both KL-repaired spectra and the previously discussed eigenspectra, it can be concluded that the convergence of the eigenspectra implies the convergence of the repaired spectrum, and vise versa. Furthermore, the quality of a repaired spectrum should not depend on the initial gap-interpolation technique. Figure 3 shows the KL-repaired spectra, using linear- and meaninterpolations for the initial gap approximation, $m=10$ and at $i=20$. The two are shown to be very similar to each other. The insert shows the corresponding $\operatorname{Tr}\left(f^{R}(m) f^{0} f^{R}(m)\right)$ as a function of iteration. The convergence behavior seems different in both cases, nevertheless they are approaching each other with a difference in the actual value about $0.3 \%$ which is small as can be seen in the plots of the spectra in the main graph. This is a desired result, because if the whole formalism is robust, the repaired spectrum should 
not be different due to different procedures used in the initial gap-fixing.

\subsection{The Effect of Sample Size}

Another important aspect of the KL-eigenspectra construction is the number of observed spectra necessary as the input. In principal, as we increase the number of galaxy spectra, a more representative and general set of eigenspectra should result. The question remains, however, how much more generality would be gained by including more observed spectra in the analysis? Fundamentally, does there exist a minimum number of input galaxy spectra such that the eigenspectra set start to converge? This is important because we can thus use a minimum number of randomlychosen observed spectra in the survey to derive a set of eigenspectra which nevertheless contain all the necessary information within the full data set. Figure 4 shows an attempt to answer this question. In these figures, the commonality percentages of two subspaces spanned by (a) 2 (b) 3 and (c) 10 eigenspectra are plotted versus the number of galaxy spectra $\mathrm{N}_{(h)}$ used in the sample. The commonality is similar to that previously discussed for the trace quantities except here we compare the set of eigenspectra derived from $\mathrm{N}_{(h)}$ input galaxy spectra with that from a smaller number of spectra $\mathrm{N}_{(h-1)}$. This is defined as follows

$\operatorname{commonality}(\%) \equiv \frac{\operatorname{Tr}\left(e\left(N_{(h-1)}\right) e\left(N_{(h)}\right) e\left(N_{(h-1)}\right)\right)}{D_{e}} \times 1$

where $e\left(N_{(h)}\right)$ is the sum of projectors of the subspace spanned by $D_{e}$ eigenspectra, derived from $N_{(h)}$ galaxy spectra, using $m=D_{e}$ eigenspectra for gap-repairing. The number of iterations for the gap-correction is 20 . The smallest number of spectra we consider is $139\left(=\mathrm{N}_{(0)}\right)$ and the largest number 40044. The galaxies in each case are randomly selected from the full SDSS sample.

For all cases, convergent trends are present as more spectra are included. For the case of two eigenspectra (Figure 4a) we can see that only about 500 galaxy spectra are needed in order to construct the first two eigenspectra to an $0.5 \%$ accuracy when compared to the final converged set. The inclusion of the third mode (Figure $4 \mathrm{~b})$ requires more spectra to obtain a similar accuracy though the convergence behavior is

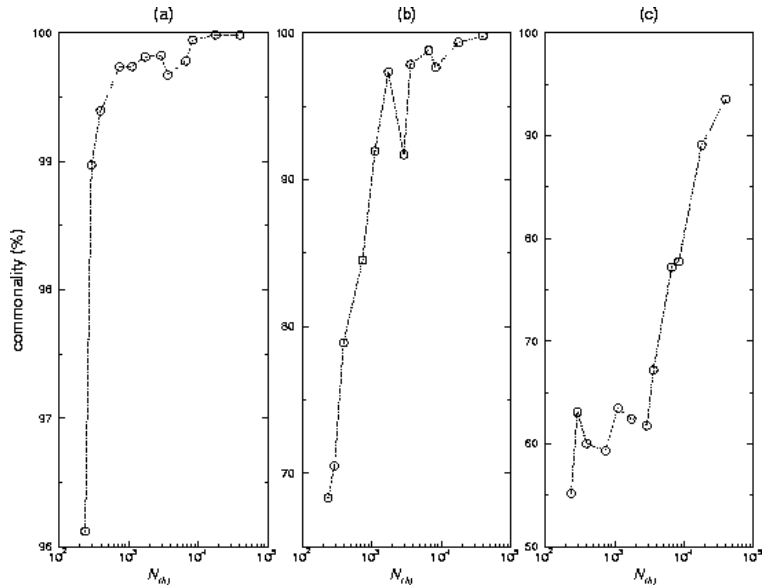

Fig. 4.- The commonality measurement of the subspaces formed by the set of eigenspectra derived using different numbers of observed galaxy spectra where the subspaces are spanned by the first (a) 2 (b) 3 and (c) 10 eigenspectra respectively. The results show that the eigenspectra set converge as a function of learning-set size in the KL gap-correction formalism.

very similar to (a) (with slightly larger fluctuations). Nevertheless, only about 1000 galaxy spectra are needed for $90 \%$ commonality. These results are consistent with the fact that most types of galaxy spectra can be described with 2 to 3 colors (Connolly et al. 1995) and therefore a random sampling of a few thousand galaxies can be expected to cover the full color distribution for these galaxies.

In Figure (c), it is interesting that the convergent behavior is different from that in (a) and (b). With a sample size smaller than about 3000 to 4000 , the improvement in the set of 10 eigenspectra is small. However, once the number of spectra used exceeds that threshold, the convergent rate dramatically increases. This finding suggests that there exists a minimum number of galaxy spectra that we need to include in our KL analysis in order to fully sample the true distribution of galaxy spectra. Combining this with the fact that the higher-order modes in the eigenspectra tend to correspond to spectral features in galaxies with prominent emission lines (this will be discussed in detail in Section 5) and the fact that those galaxies only comprise about $0.1 \%$ of the whole sample, the behavior in (c) can be understood as the effect of 
including galaxies with relatively extreme spectral properties. When we randomly pick about 1000 galaxies from the sample there are a few emission line galaxies. As more spectra are included we eventually reach a threshold where we begin to sample the extreme emission line galaxies. Once we have included a small number of these emission line galaxies (with a sample size of $3000-4000$ galaxies we would expect three to four emission line galaxies) the information they contain is now incorporated within the KL eigenbases. The dramatic increase in the convergence rate come from the fact that, while rare, these extreme emission line galaxies can still be described by a handful of spectral components (i.e., once we have a small number of them in the sample we can map out their full distribution).

To conclude, there exist a minimum number of galaxy spectra we need to observe in order to derive a convergent set of eigenspectra. Approximately $10^{4}$ spectra are sufficient for a $90 \%$ convergence level with ten eigen-components (Figure 4c). This is sufficient to characterize the spectral types of $99.9 \%$ of galaxies within the local universe. These results are, however, purely empirical, based on randomly selecting spectra from the current data set. Thus, there is no concrete evidence to support the present result that $10^{4}$ galaxy spectra are all we need in deriving the most complete set of eigenspectra. There may exist populations of galaxies that comprise much less that $0.1 \%$ of the full galaxy sample that our current analysis is not sensitive to. In general, for a larger data set (e.g., at the completion of the survey), new galaxy types, if any, may call for more spectra to be included when constructing the eigenspectra.

\section{KL Eigenspectra and $\left(\phi_{K L}, \theta_{K L}\right)$-Classification}

The first $10 \mathrm{KL}$ eigenspectra of the 170,000 SDSS galaxies are shown in Figure 5, derived from 20 iterations and using 10 eigenspectra for gap repairing. The eigenspectra are publicly available (from the website http://www.sdss.org). The first eigenspectrum is the mean of all galaxy spectra in our sample. The continuum is similar to a Sb-type ${ }^{1}$. As we would expect from the mean

\footnotetext{
${ }^{1}$ In our work, the red- and blue-types are determined from the spectral information in the galaxies. Thus, the conventional morphological-type nomenclatures "early", "late",
}

of all spectra, nebular lines and other emission lines, as well as absorption lines such as $\mathrm{Ca} \mathrm{H}$ and $\mathrm{Ca} \mathrm{K}$, are present within this spectrum. The second eigenspectrum has one zero crossing, positive toward longer wavelengths, at around $5200 \AA$, which marks the wavelength at which the modulation in the continuum level relative to the 1st eigenspectrum occurs. In the third component, there is a zero crossing in the continuum, negative toward longer wavelengths, at around $6000 \AA$. The higher the order of the eigenspectrum, the larger the number of zero crossings which in turn adds high-frequency features to the final spectrum as these higher order modes are added or subtracted. In the higher-order modes, the eigenspectra are dominated by emission and absorption lines because each of these eigenspectra comprises emission and absorption lines plus a small fluctuation of the continuum level around zero. We illustrate this point later in the paper.

Statistically, the amount of information contained in each eigenspectrum is given by the eigenvalue of the correlation matrix of that particular mode. Table 2 lists the weights of the first $m$-modes of eigenspectra, where the weights are normalized to unity. We find that the first three eigenspectra contain more than $98 \%$ of the total variance of the data set.

It is known that there is a one-parameter description of galaxy spectra which correlates with the spectral type of a galaxy (Connolly et al. 1995). This parameter, $\phi_{K L}$, is the mixing angle of the first and second eigencoefficients. Explicitly,

$$
\phi_{K L}=\tan ^{-1}\left(a_{2} / a_{1}\right)
$$

where $a_{1}$ and $a_{2}$ are the eigencoefficients of the first and second modes of a galaxy respectively. Furthermore, the inclusion of the third component discriminates the post-starburst activity (Connolly et al. 1995; Castander et al. 2001). To follow this classification scheme, we define here

$$
\theta_{K L}=\cos ^{-1}\left(a_{3}\right)
$$

where $a_{3}$ is the third eigencoefficient. Here we

and E, S0, types etc. used in this paper are referring to spectral features which usually would have be seen in the corresponding morphological types, as suggested in Kennicutt's Atlas and other studies. 


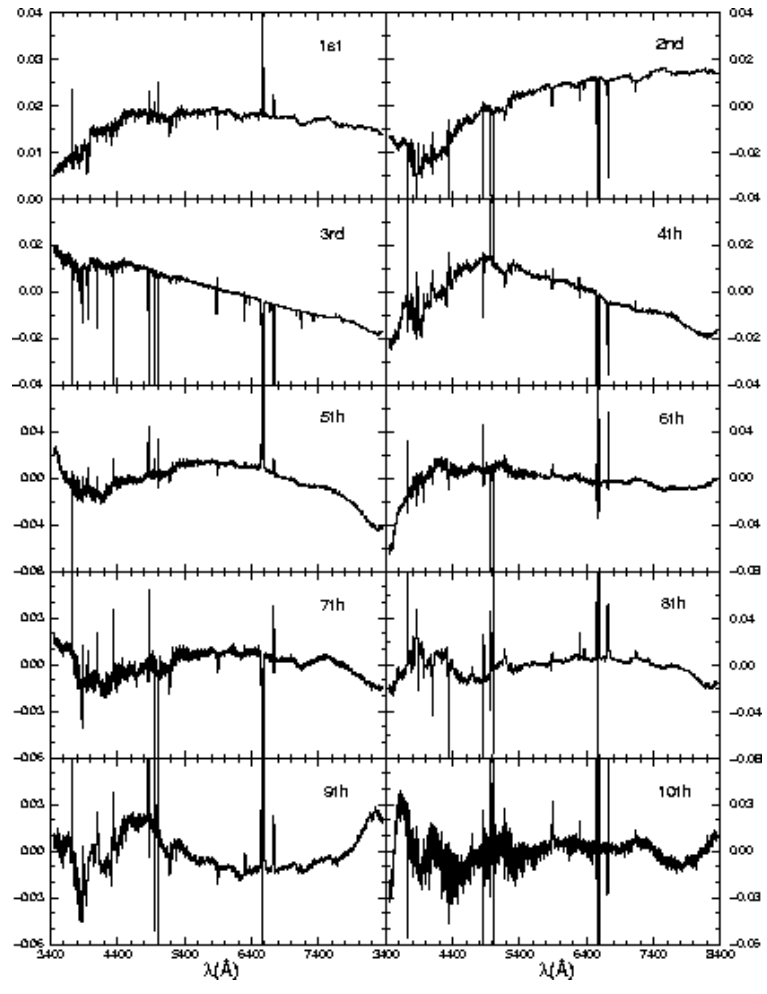

Fig. 5. - The first $10 \mathrm{KL}$ eigenspectra of $\approx 170,000$ galaxy spectra in the SDSS. Gap correction is implemented for 20 iterations.

adopt the normalization

$$
\sum_{k=1}^{10} a_{k}^{2}=1 .
$$

The first three eigencoefficients of the whole sample are plotted in Figures 6 and 7 in the forms of $a_{2}$ versus $a_{1}$ and $a_{3}$ versus $a_{2}$. More than $99 \%$ of the total galaxy population is located on the locus in Figures 6, in which the second eigencoefficients have values from $\approx 0.25$ to -0.75 . The appearance of this locus is very similar to previous works (Connolly et al. 1995). Red galaxies have positive, and relatively large second eigencoefficients, while blue galaxies have smaller, or in some cases negative values. From Figure 7 we clearly see that by introducing the third eigen-component, there is a group of galaxies being separated out from the main group. These galaxies, with negative $a_{3}$ and negative $a_{2}$ values, exhibit post-starburst activity in their spectra. A much smaller group (about $0.1 \%$ ) with positive $a_{3}$ and negative $a_{2}$ values is

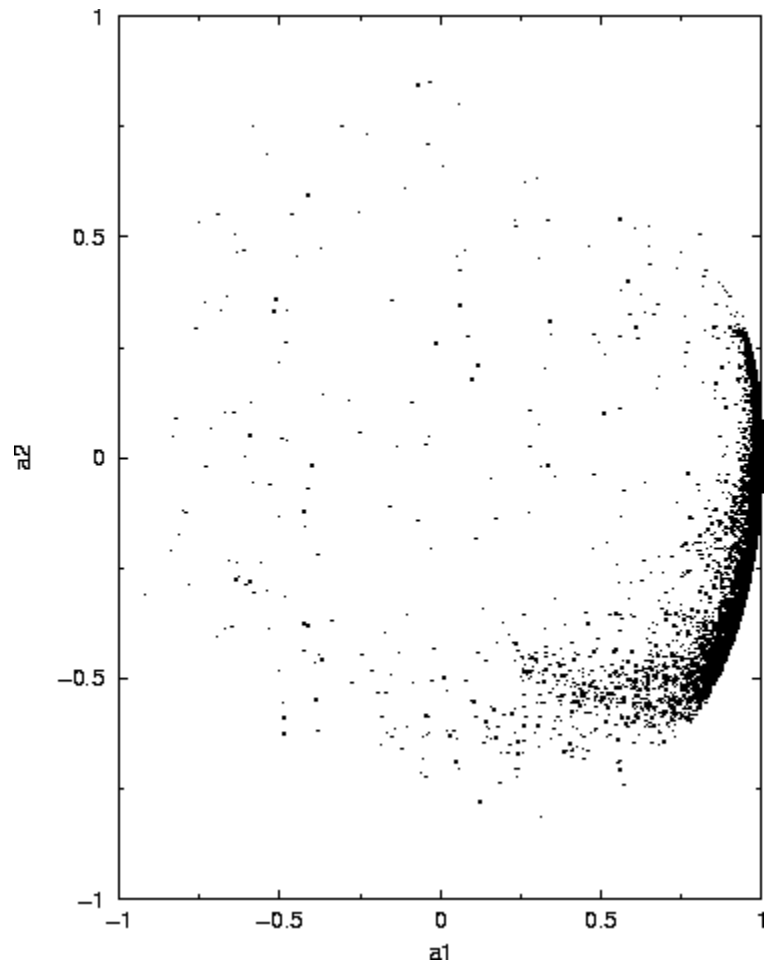

Fig. 6.- Eigencoefficients $a_{2}$ versus $a_{1}$ of our sample ( $\approx 170,000$ galaxy spectra). More than $90 \%$ of the whole galaxy population are located on this main locus. The trend is similar to that in previous works (Connolly et al. 1995), with red galaxies having larger, positive $a_{2}$ values and blue galaxies having smaller, or negative values.

also seen. These are outliers and will be discussed later. The resulting $\phi_{K L}$ versus $\theta_{K L}$ is plotted in Figure 8 for all galaxies in the sample excluding those galaxies with $a_{1}<0$ (118 objects). These $a_{1}<0$ sources tend to be either artifacts within the data (M. SubbaRao, private communication) or spectra that are not visually confirmed as a galaxy spectrum. In Figure 8a, the sequence from red to blue to extreme emission line galaxies is illustrated. The boxes drawn show the regions from which a set of spectral types are identified. They range from the early-type at the top of the plot to emission line galaxies at the bottom. The spectra for these subsamples are shown in Figure 9(a-f), ranging from red to emission line galaxies. The spectral energy distributions shown are the mean of all the observed spectra classified to be in the range $\left(\phi_{K L}^{s}, \phi_{K L}^{e}, \theta_{K L}^{s}, \theta_{K L}^{e}\right)$, where the super- 


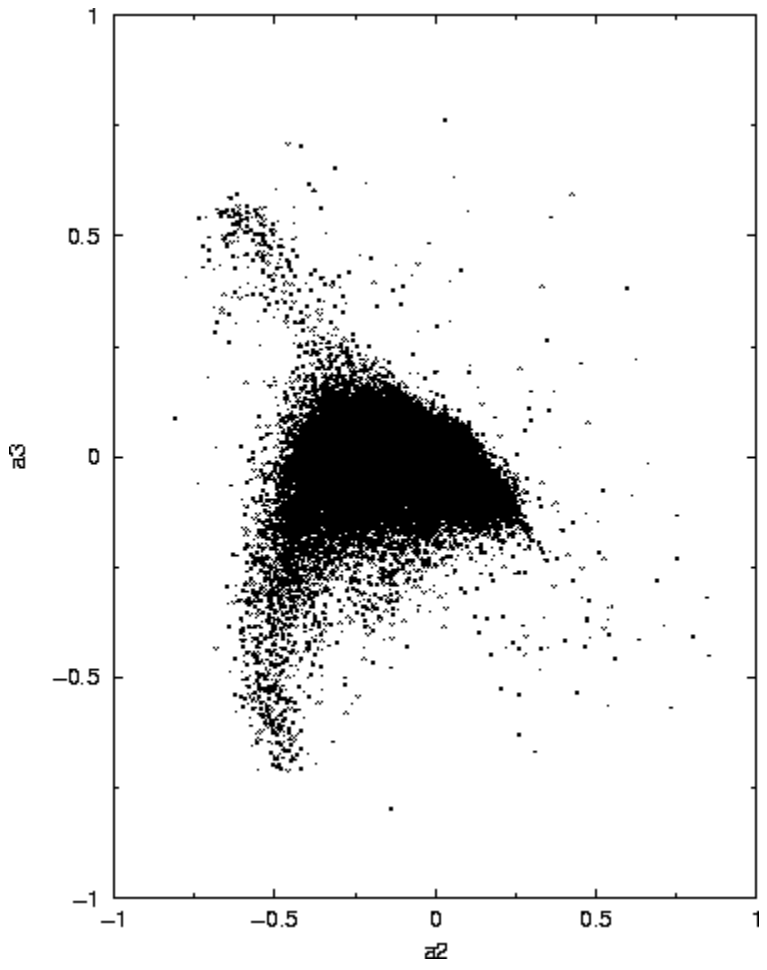

Fig. 7.- Eigencoefficients $a_{3}$ versus $a_{2}$ of our sample $(\approx 170,000$ galaxy spectra). The introduction of the third eigencoefficient further discriminates galaxies with post-starburst activity (they are the group of galaxies with negative $a_{3}$ and $a_{2}$ values in this plot). Also, a group of outliers are apparent (a small group of objects with positive $a_{3}$ and negative $a_{2}$ values) which are explained in the text.

scripts $^{s}$ and ${ }^{e}$ denote the starting and ending values bounding the range. The actual values are chosen such that the resultant mean spectra agree with the galaxy spectra of each type in Kennicutt's atlas of nearby galaxy spectra (Kennicutt 1992). The flux levels are in good agreement with those in the atlas, which leads us to believe that the classification is physically sound as well as having statistical rigor. Spectra with similar spectral features are therefore seen to be clustered by the KL procedure. Due to the smoothing of spectral inhomogeneities with a large number of galaxies, the resultant mean spectra have very high signal-tonoise levels. This result demonstrates the power of the KL transform for calculating mean (or composite) spectra (e.g., Eisenstein et al. 2003 for the

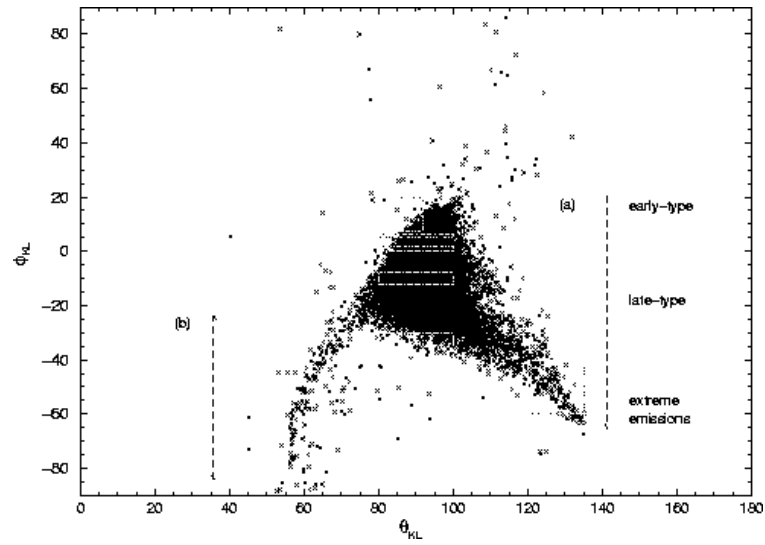

Fig. 8.- $\left(\phi_{K L}, \theta_{K L}\right)$-classification of $\approx 170,000$ SDSS galaxy spectra. (a) illustrates the sequence along which the galaxy spectral types are identified. (b) Outliers, mostly spectra without significant spectral features. The angles are in degrees. Most outliers have large errors in their redshift estimations, while $90 \%$ have low signal-to-noise ratios. The boxes are areas in which the mean of all of the observed spectra correspond to red, blue and emission line galaxies. See Figure 9 for the mean spectra.

mean spectrum of the SDSS massive galaxies).

Table 1 shows the number of observed galaxy spectra in each of the regions described previously. We stress that the sum of all galaxies listed in the table is not equal to the total number of galaxies in the data set because the ranges chosen comprise a subset of the full $\left(\phi_{K L}, \theta_{K L}\right)$-plane. The early late- to intermediate late-types galaxies (with $-12^{\circ}<\phi_{K L}<5^{\circ}, 80^{\circ}<\theta_{K L}<100^{\circ}$ ) dominate within the whole data set which agrees with the well-known fact that late-type galaxies dominate the field populations in terms of number counts.

Apart from the main locus in Figure 8 the region marked "(b)" identifies a group of outliers, forming approximately $0.1 \%$ of the full sample (190 sources). These are unusual sources that arise due to artifacts within the reduction pipelines, errors within the spectra themselves or possibly due to new classes of astrophysical sources. In later processing runs (idlspec2D v4.9.8, as of 13 th of August, 2002) only 68 of these sources remain in the main galaxy sample. Approximately half of them have the ZWARNING flag set to 4 , which 

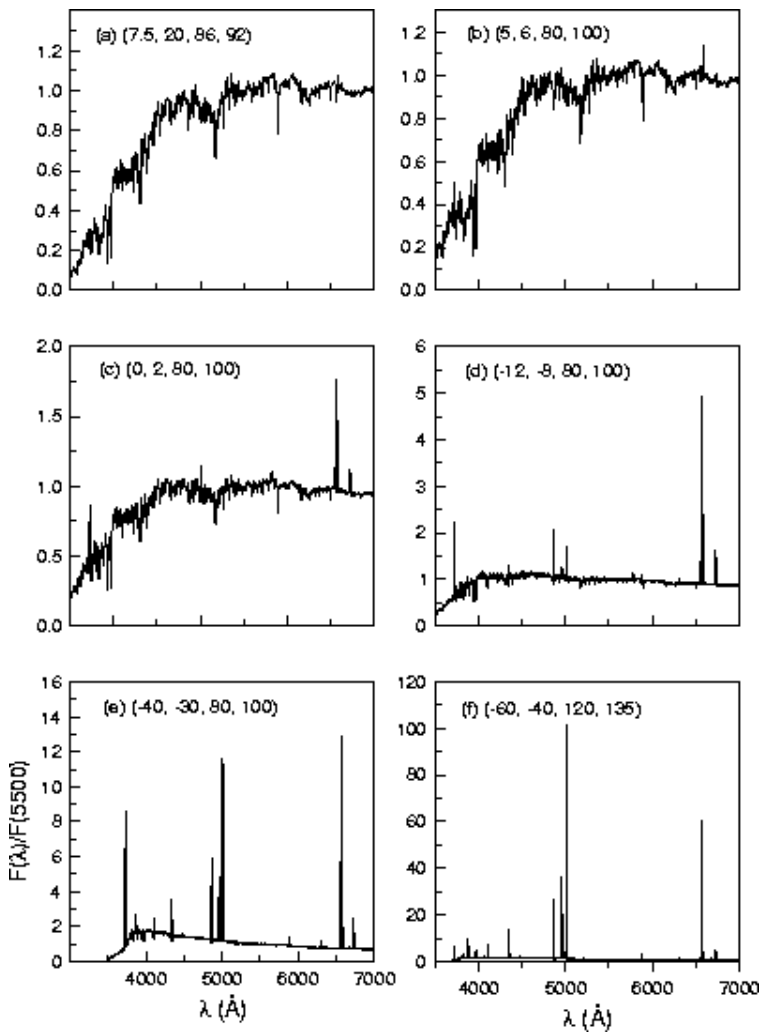

Fig. 9.- Mean of all the observed spectra in different ranges of $\left(\phi_{K L}^{s}, \phi_{K L}^{e}, \theta_{K L}^{s}, \theta_{K L}^{e}\right)$, with the classification angles being (a) $(7.5,20,86,92)$, (b) $(5,6,80,100)$, (c) $(0,2,80,100),(d)(-12,-8,80$, $100)$, (e) $(-40,-30,80,100)$ and (f) $(-60,-40,120$, 135) are shown.

indicates that there are large errors in the redshift estimations. This results in less than $0.02 \%$ of the spectroscopic sample having spectra that can be considered unphysical (a testament to the remarkable accuracy and performance of the current spectroscopic reduction pipelines). Considering all of these sources as a whole $90 \%$ have signal-tonoise ratios $(\mathrm{S} / \mathrm{N})$ higher than the mean survey quality (the $\langle S / N\rangle$ is 15.9 in the data set).

Of the remaining 30 galaxies within this outlier class, most have relatively high redshifts $(z \sim$ $0.2-0.5)$ as assigned by the pipelines. Some of these sources classed as galaxies by the pipelines do not appear to be galaxy spectra when inspected visually. On the other hand, for those that are galaxies as inspected by us, we found that the pipelines have assigned incorrect redshifts to some

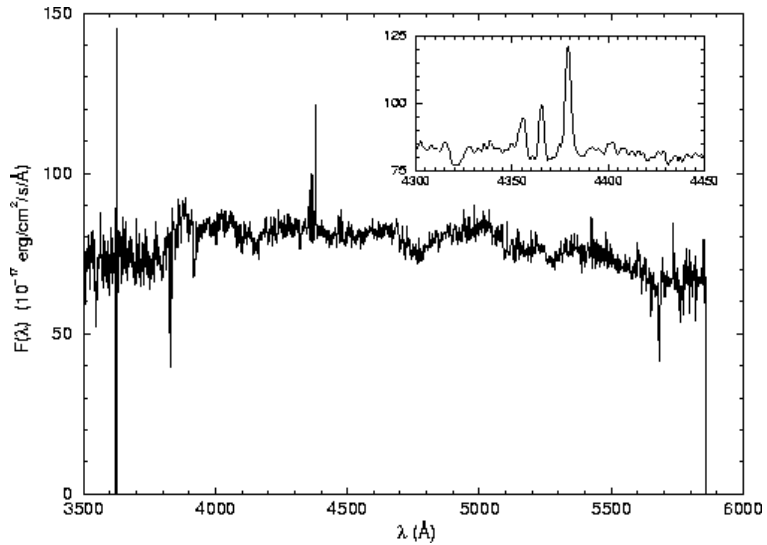

Fig. 10. - One of the outliers in the $\left(\phi_{K L}, \theta_{K L}\right)$ plane. The redshift of this galaxy is incorrectly assigned by the spectroscopic pipeline.

of these spectra. As expected, the gap-repairing procedure fails in those objects and the resulting expansion coefficients have unphysical values. An example is shown in Figure 10, according to the assigned redshift, this object has a redshift 0.5394 , which is obviously incorrect from the locations of $\mathrm{N}$ II $+\mathrm{H} \alpha+\mathrm{N}$ II lines, as shown in the insert (this galaxy should have a redshift of 0.0236 ). The outcome is that the magnitudes of the 2nd and the 3rd eigencoefficients obtained by a KL of all the objects in this group are roughly the same but with different signs, meaning that no lines that are representative of typical spectral types are found. This result suggests that KL technique is a powerful tool for identifying artifacts within any spectral reduction procedure.

The above results show that the classification is successful in allowing the galaxy types to be identified using the first three eigencoefficients and that it may serve as a way for error checking. How do the eigenspectra actually perform in reconstructing the spectra? Figure 11(a-f) shows, for the same range of $\left(\phi_{K L}^{s}, \phi_{K L}^{e}, \theta_{K L}^{s}, \theta_{K L}^{e}\right)$ as above, the means of all KL-reconstructed spectra. A KLreconstructed spectrum, using $m$-eigenspectra, is given by

$$
f^{R}(m ; \lambda)=\sum_{k=1}^{m} a_{k} e_{k}(\lambda) .
$$

It should be noted that the KL-reconstructed spectrum is different from the KL-repaired spectrum we mentioned previously (in that case the 

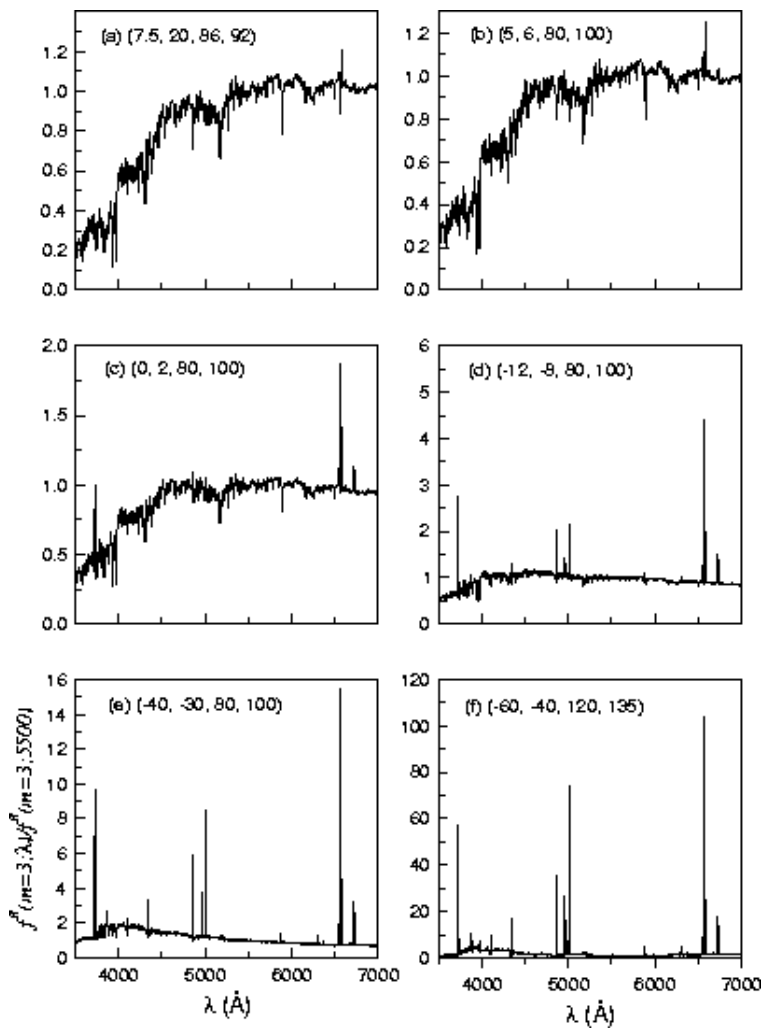

Fig. 11. - The means of all KLreconstructed spectra (a-f) in different ranges of $\left(\phi_{K L}^{s}, \phi_{K L}^{e}, \theta_{K L}^{s}, \theta_{K L}^{e}\right)$ (the bounding boxes are the same as those in Figure 9). The first three eigenspectra are used in the reconstruction. The continua and most of the line features are in excellent agreement with those of the mean spectra shown in Figure 9.

repairing is in the gap regions only). For convenience, the mean of all KL-reconstructed spectra in a given range is abbreviated as "KLreconstructed spectrum" in the following sections unless otherwise specified. Comparing the mean spectra in Figure 9(a-f) with the reconstructed ones in Figure 11(a-f) (3 modes are used), the continuum levels and most emission lines are in excellent agreement with the mean spectra (except for the galaxies with extreme emissions, which we will discuss later). These results are consistent with previous claims that two eigenspectra are enough to describe most of the spectral types in galaxies (Connolly et al. 1995). Our present classification scheme of using two mixing angles of the first three eigencoefficients is also justified.
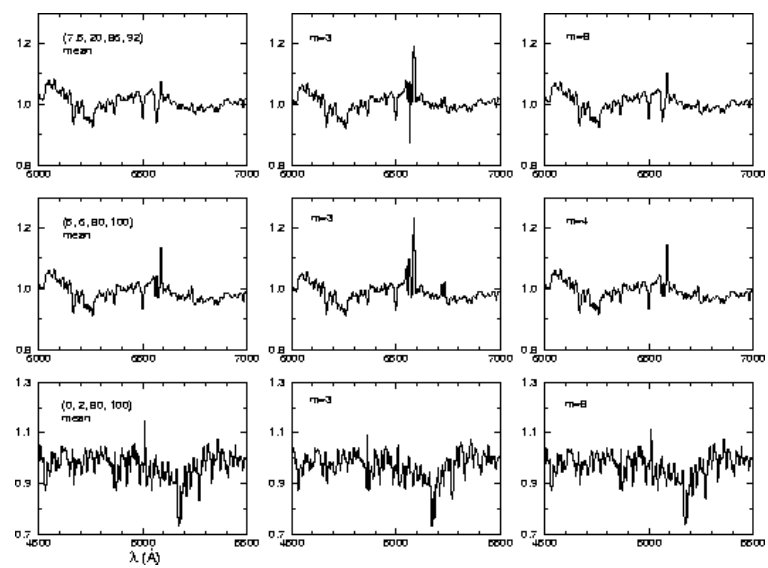

Fig. 12.- Number of modes needed to reconstruct some of the lines in early-type to early late-type galaxy, with the classification angles in the ranges $(7.5,20,86,92)$ (top panel), $(5,6,80,100)$ (middle panel) and $(0,2,80,100)$ (bottom panel). The figures on the leftmost panels show the mean spectra in each type, and the consecutive figures show the KL-reconstructed spectra with different numbers of modes. All spectra are normalized at $5500 \AA$.

The 3-mode KL-reconstructed spectra shown in Figure 11(a-f) also suggest that to reconstruct some of the lines and line ratios, more eigenspectra are necessary. Figure 12 shows in detail the emission lines that require more than 3 modes for reconstruction. These figures show early-type to late-type galaxies (from top to bottom). With the first eight eigenspectra, the amplitude of the N II line for galaxies with classification angles in the range $(7.5,20,86,92)$ can be correctly recovered. Similarly, the first four modes are sufficient for the $\mathrm{N}$ II and $\mathrm{H} \alpha$ reconstruction for galaxies with classification angles in the range $(5,6,80,100)$. Progressing to bluer galaxies with classification angles in the range $(0,2,80,100)$, the first eight modes are enough for the O III reconstruction. Similarly, Figure 13 shows the cases for galaxies with prominent emission features. We find that the first four modes are enough to reconstruct the amplitudes and line-ratios O III[5008.240]/O III[4960.295] and $\mathrm{H} \beta[4862.68] / \mathrm{O} \mathrm{III}[4960.295]$ for those with classification angles in the range $(-12,-8,80,100)$. For galaxies in the range $(-60,-40,120,135)$, the lineratio $\mathrm{N}$ II[6585.27]/N II[6549.86] is correct with three eigenspectra, while the first eight modes are 
enough to further retrieve the amplitudes of the two N II lines. The maximum differences between the amplitudes of the mean and the reconstructed lines (which we define as the error of the reconstruction of a particular line) in the abovementioned cases are about $10 \%$.
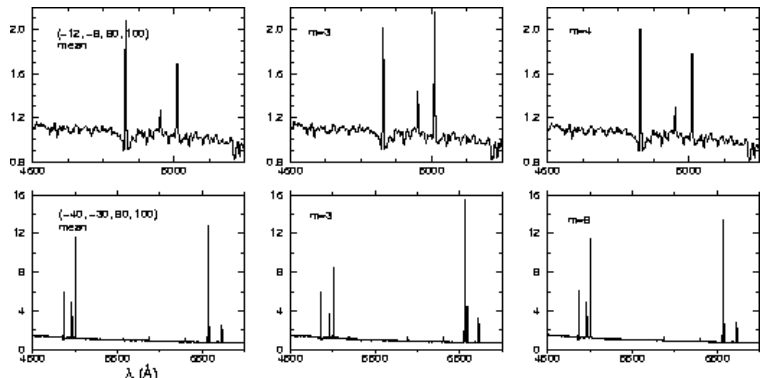

Fig. 13. - Same as Figure 12, but for galaxies with the classification angles in the ranges $(-12,-8,80$, 100) (top panel) and $(-40,-30,80,100)$ (bottom panel).

Therefore, for all but the most extreme emission line galaxies, eight spectral components, or modes, are sufficient to reconstruct the spectral line ratios to an accuracy of about $10 \%$ (a factor of 500 in compression of information within the galaxy spectra). For the reconstruction of galaxies with extreme emissions, however, the performance is not satisfactory when using a small number of eigenspectra. Nevertheless, ten eigenspectra are sufficient to recover the continuum level (see the mean and KL-reconstructed spectra in the enlarged continuum region). The residuals of the mean spectra and the KL-reconstructed spectra are shown in Figure 14, where (a-d) correspond to the reconstructions with $3,4,5$ and 10 eigenspectra respectively. There are substantial improvements in using ten eigenspectra, especially in nebular lines and S II lines, and various line ratios. The typical errors in the fluxes of lines remains around $15-25 \%$.

This is not a surprising result. On one hand, the result follows because of the increasingly dominant role of lines in the higher-order modes compared with the continuum. On the other hand, statistics also play a factor. The early and intermediatetype galaxies dominate the population of galaxies while emission and extreme emission line galaxies comprise just a few percent of the total population. Thus, galaxies with significant emission call for more eigenspectra and higher-order modes in their reconstructions. Besides the statistical reasons, the inevitable variations in line-widths of emission lines make it comparatively difficult to reconstruct them accurately using linear combinations of eigenspectra.

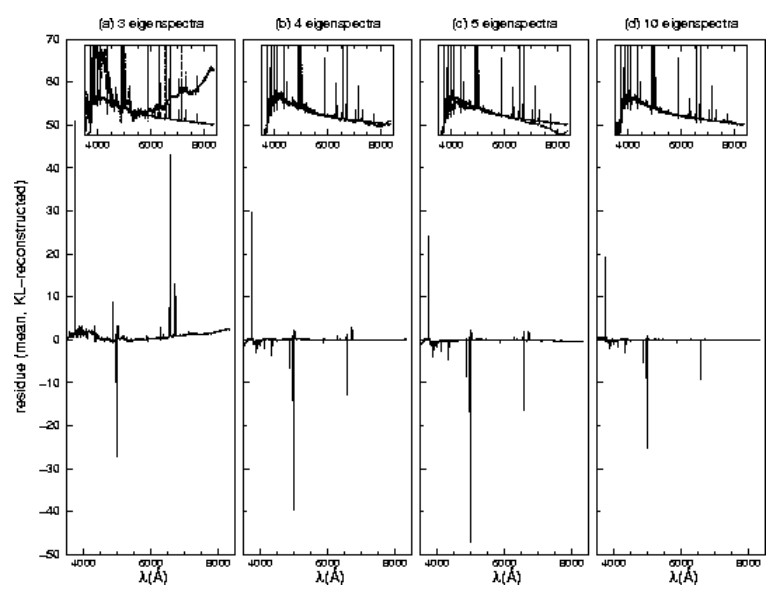

Fig. 14.- The residuals of the mean spectrum of extreme-emission galaxies (classification angles in the range $(-60,-40,120,135))$ with the KLreconstructed spectrum using (a) 3, (b) 4, (c) 5, and (d) 10 eigenspectra. The inserts are the enlarged regions of the continuum levels, in each case the solid line is the mean spectrum and the dotted line is the mean of the KL-reconstructed spectra. The spectra are normalized at $5500 \AA$.

Due to the fact that the spectral features in extreme emission line galaxies are distinct from other types of galaxies, they still reveal themselves in the plane $\left(\phi_{K L}, \theta_{K L}\right)$. Thus, for the main purpose of this work, which is obtaining a robust and objective classification of galaxies, the less satisfactory performance of reconstructing some emission lines in galaxies with extreme emission lines does not have a significant effect. However, if detailed diagnosis of lines (for example, the flux-ratio of two lines) in those galaxies are of interest, then more modes are needed. Better yet, a separate analysis using KL with those emission line galaxies is suggested.

\subsection{KL-reconstruction as Low-pass Filter- ing}

The inclusion of all the modes in the KLreconstruction of a given spectrum should, in prin- 
ciple, reproduce all spectral features (including the noise). As higher-order modes contain higher frequency signals and smaller variances of the sample, the inclusion of only a few lower-order modes would thus suppress the noise present in the spectrum. Examples of the comparison between the observed spectra and the KL-reconstructed ones are shown in Figure 15(a-c). In each case, the spectrum is reconstructed with the first 10 eigenspectra, and normalized at $5500 \AA$. From these noise-free reconstructed spectra, it becomes a simple task to identify and classify the spectral lines.
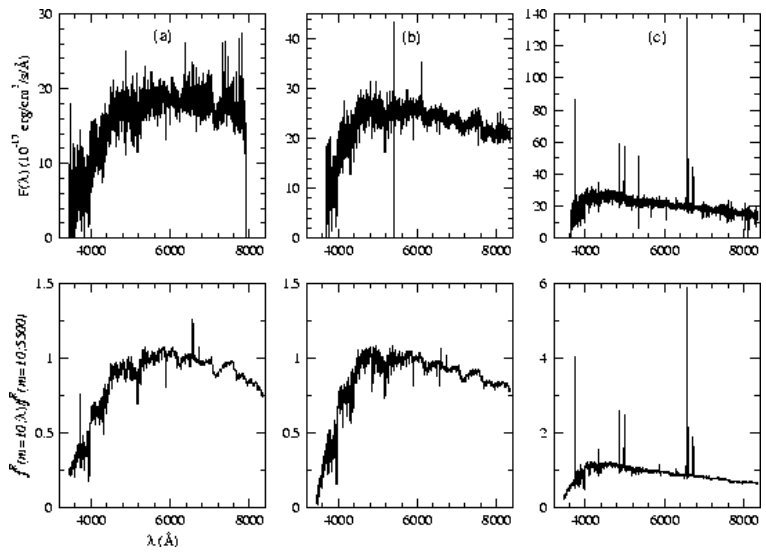

Fig. 15.- Low-pass filtering of the observed spectra (a-c) by the KL-reconstruction. The figures on the lower panel show the KL-reconstructed spectra respectively for each observed spectrum (normalized at $5500 \AA)$.

\section{Reliability of the Galaxy Classification}

Any classification scheme has to be repeatable in order to be useful. If we measure the spectrum of a galaxy on different nights in different conditions, would the classification still be the same? To answer this question, we are fortunate in that many galaxy spectra in the SDSS data set are taken on multiple nights (Blanton et al. 2002). A total of 1,854 galaxies were found in our sample to be not unique (i.e., they have been observed and reduced independently). A further thirty thousand galaxies were found in the SDSS spectroscopic data to have been observed on multiple nights with different observing conditions (often these individual observations do not meet the signal-to-noise requirements of the SDSS spectral observations). The quantitative interpretation of the repeatability of classifications based on these plates may be difficult due to the variation in signal-to-noise ratios. Nevertheless all repeat observations are selected for this part of our work.
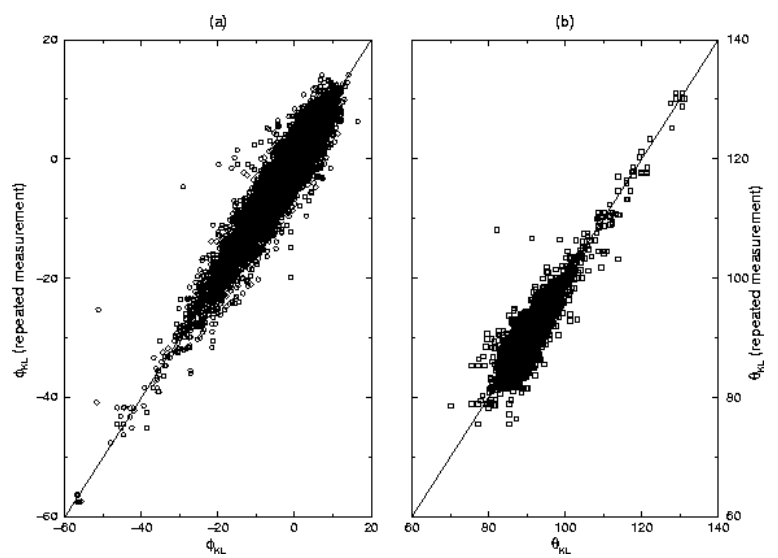

Fig. 16.- Reliability of the KL classification of galaxies. The classification parameter of each object is plotted against that of the repeated measurement, for the cases (a) $\phi_{K L}$ and (a) $\theta_{K L}$.

Of the thirty-thousand sources, only those with flags PRIMTARGET, OBJTYPE and CLASS equal GALAXY are selected (together with the requirement that all sources are present in the most up to date reductions). This selection results in thirteen-thousand objects in the final sample. Figure 16a and Figure 16b show a comparison between the $\phi_{K L}$ and $\theta_{K L}$ values assigned by our classification scheme to those galaxies with the highest signal-to-noise and the classification derived from the repeat observations. The solid line corresponds to the location of the one-to-one correspondence between the two measures. The dispersions in $\phi_{K L}$ and $\theta_{K L}$ are $2.35^{\circ}$ and $1.61^{\circ}$ respectively. This agreement is excellent, as these angle dispersions correspond to small changes in the resulting repaired spectra. The agreement also spans a large range in both classification angles. The implication of this finding is that a truly reliable and repeatable classification scheme is obtained which validates the repeatability of the spectrophotometry of the SDSS.

In order to determine a representative signal-tonoise ratio for each spectrum the median signalto-noise ratio is adopted (the flag SN_MEDIAN in spZbest-plate-mjd.fits). The dependence of the 
rms error in the measured angles on the signal-tonoise of the observations (where the signal-to-noise is selected to be the lower one of any pair of observations) is shown in Figure 17. From the current data set we observe a weak trend with larger discrepancies in the classification for those observations with lower signal-to-noise ratios. The mean (absolute) discrepancies in the classification angles $\left(<\left|\delta\left(\phi_{K L}\right)\right|>\right.$ and $<\left|\delta\left(\theta_{K L}\right)\right|>$ ) and mean signal-to-noise ratios are calculated in the ranges of signal-to-noise ratio $(0.0-10.0),(10.0-15.0)$, $(15.0-20.0),(20.0-30.0)$ and larger than 30.0. The dependence is very similar in both the $\phi_{K L}$ and $\theta_{K L}$ angles. The error bars are set by the rootmean-square fluctuations in both quantities. The vertical line marks the calculated mean signal-tonoise ratio of all the galaxies defined as meeting the survey quality (a signal-to-noise of 15.9).

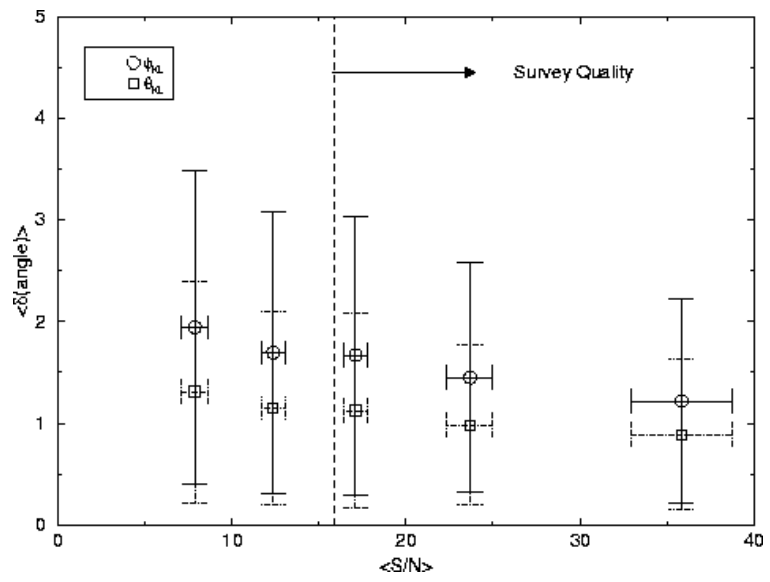

Fig. 17. - The mean discrepancy in the classification angles derived from the KL analysis (circles are $<\left|\delta \phi_{K L}\right|>$ and squares, $<\left|\delta \theta_{K L}\right|>$ ) versus the mean signal-to-noise ratio of all spectra. All spectra in this plot have been observed more than once.

For those sources meeting the survey quality signal-to-noise criteria, the maximum errors in the two mixing angles are, approximately, three degrees in $\phi_{K L}$ and two degrees in $\theta_{K L}$. This result shows that the classifications based on the SDSS spectra are repeatable and robust to the variable signal-to-noise within the spectroscopic data. The fact that the signal-to-noise dependence is weak suggests that the noise within the spectra are essentially Poisson such that the projection of a noisy spectrum does not add substan- tial artifacts into the expansion coefficients. Despite this weak dependence in the distribution of expansion coefficients with signal-to-noise we do find instances where the spectral properties of the galaxies change between pairs of observations. For example, in one case we find that the strength of the O II lines change by about $20 \%$ between two separate observations. It is not clear whether this difference is due to a calibration error or due to variability in the source.

\section{A Simple Model of the Distribution of Galaxy Populations}

From studies of the luminosity function of galaxies it has been shown that the distribution of galaxies comprise a number of populations or classes. It is, therefore, natural to ask how many classes are present within the SDSS spectroscopic data and how many galaxies occupy each class. We plot in Figure 18 the frequency distribution of $\phi_{K L}$, (for the moment we neglect $\theta_{K L}$ because the extreme emission line galaxies contribute less than a percent to the full galaxy distribution). The bin width is $\phi_{K L}=0.5^{\circ}$ and the histogram is normalized to unity. Visually, there appear to be two to three dominant "classes" or subtypes within the $\phi_{K L}$-distribution. To further investigate the number of subpopulations, we adopt the Akaike Information Criterion (AIC). AIC is widely used in model selection in a number of different disciplines. The details of AIC and its application in astronomy can be found in Connolly et al. (2000). Basically, in AIC, a score is assigned to the model distribution, allowing a quantitative comparison with the true distribution of the data. Naturally, more parameters within a model yield a better fit to the data. To counter this the AIC penalizes the score based on the number of parameters within a model. The AIC score is given by the following,

$$
\text { Score }(\mathrm{AIC}) \equiv \ln \mathcal{L}-R
$$

for a given model. In this definition, $\ln \mathcal{L}$ is the log-likelihood function, and $R$ is the number of parameters in the model. As a result, the higher is the score the better the model.

The first step in the analysis is to choose a functional form for the model; a Gaussian model is adopted here. We fit models with increasing numbers of Gaussian components to the $\phi_{K L^{-}}$ 


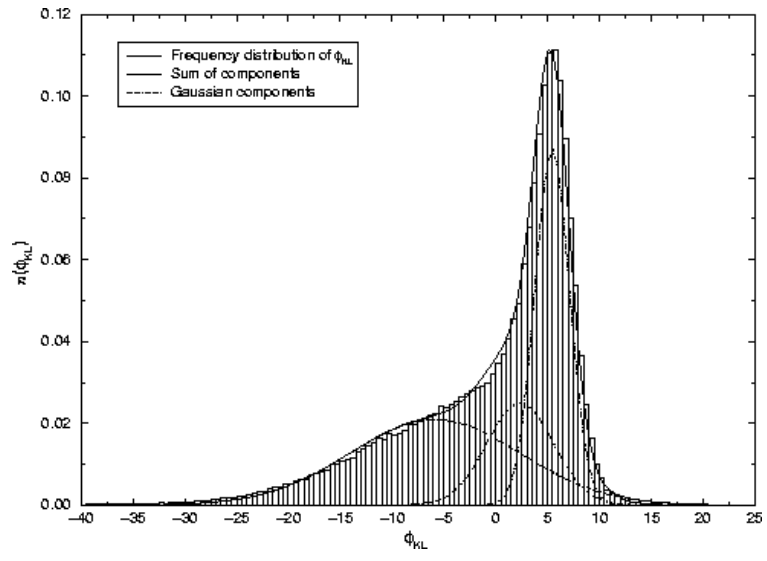

Fig. 18. - Frequency distribution of $\phi_{K L}$. The three modeled Gaussian populations are shown (dotted lines), with the peaks correspond to early late- through to intermediate late-types. The solid line is the sum of the three modeled populations.

distribution and show the resultant AIC score in Figure 19 as a function of the number of Gaussians $(n G)$ in the linear mixture model. The insert shows an enlargement of the region around $n G=2-6$. We find that for $n G=5-6$, the scores start to flatten off $(0.01 \%$ difference in the AIC score), whereas the major improvement occurs at $n G=2$. In a statistical sense, $n G=5-6$ give the best score and therefore it would appear that at most six subgroups might contribute to the distribution of the $\phi_{K L}$ values. We do note, however, that there is no underlying physical motivation for assuming a Gaussian mixture model and that as the number of Gaussians in the model exceeds four the individual Gaussian contain no direct physical meaning. That is to say, the individual populations for $n G>4$ actually overlap, forming redundant descriptions. Thus, we estimate that a linear model of a mixture of three Gaussians is sufficient for modeling the populations of galaxies in our data set. The form of the model is as follows

$$
\begin{aligned}
n\left(\phi_{K L}\right)= & G(0.087,5.43,2.39)+G(0.025,2.34,4.55) \\
& +G(0.021,-5.86,11.58),
\end{aligned}
$$

where $n\left(\phi_{K L}\right)$ is the number density (normalized, $\left.\int n\left(\phi_{K L}\right) d \phi_{K L}=1\right)$ as a function of $\phi_{K L}$, and $G(C, M, S)$ is a Gaussian function of $\phi_{K L}$

$$
G(C, M, S)=C e^{-\left[\left(\phi_{K L}-M\right) / S\right]^{2}} .
$$

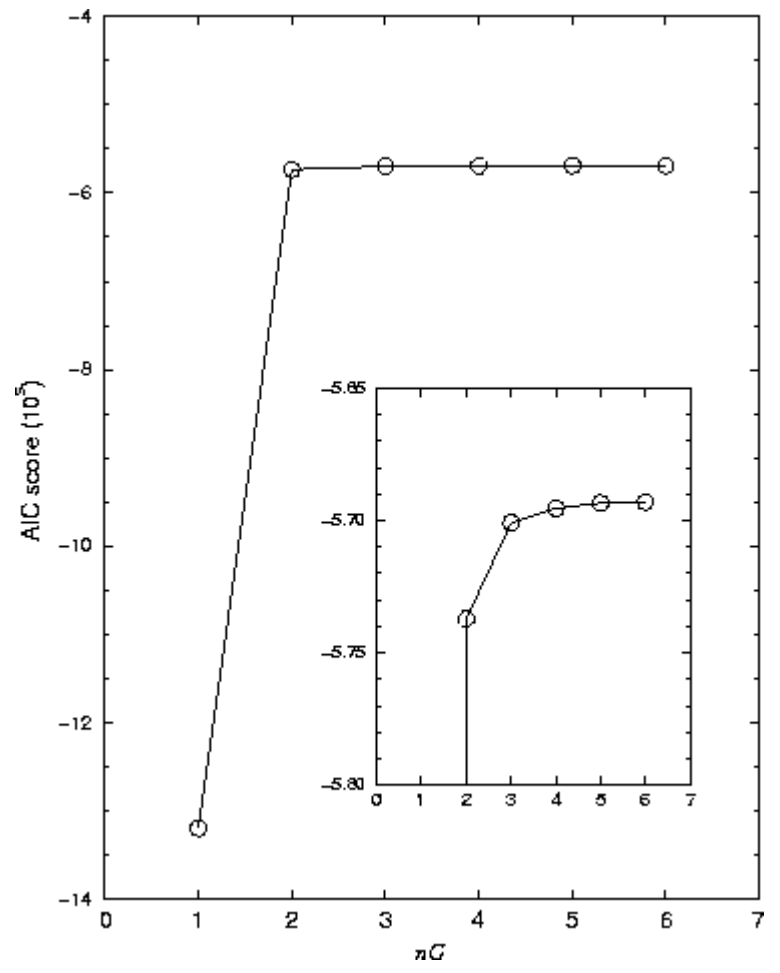

Fig. 19.- AIC score $\left(\times 10^{5}\right)$ as a function of the number of Gaussians in the population model.

The three Gaussians are illustrated by the dotted lines in Figure 18. Comparing the mean values (in $\phi_{K L}$ ) of the Gaussian distributions with the ranges of $\phi_{K L}$ over which the mean spectra of different galaxy types are derived (see Figure 9, also see Table 1), these distributions correspond approximately to early through to intermediate types. Because the first two eigenspectra, i.e., $\phi_{K L}$, roughly describe the color of a galaxy, the different sub-populations we obtain should relate to the color separation found in the SDSS EDR galaxies (Strateva et al. 2001) in which the bimodal $u^{*}-r^{*}$ color distribution corresponds to early- (E, S0 and Sa) and late- (Sb, Sc and Irr) types (Shimasaku et al. 2001). Besides, optical colors of all galaxies in the SDSS were found to be correlated very strongly with ${ }^{0.1}(g-r)$ color (i.e., the $g-r$ color for galaxies at redshift $z=$ 0.1 , which was also found to be double-peaked (Blanton et al. 2003a). 


\section{Applications of KL eigenspectra}

As we have shown, the eigencoefficients that describe a galaxy spectrum correlate strongly with its intrinsic spectral type. We will leave for a later paper a detailed investigation of the correlations inherent within the eigenbases and their relations to physical spectral energy distribution models such as Bruzual and Charlot (1993). In the following section we will just note a number of the interesting correlations present within the galaxy spectra and eigenspectra.

\subsection{Line Correlations within the Eigen- spectra}

Each galaxy spectrum can be constructed through a linear combination of the eigenspectra. While the relative weights of these combinations (i.e., the expansion coefficients) have been shown to provide a basis for the classification of the galaxy spectra, the details of the individual eigenspectra provide insight into the relative correlations between the emission and absorption lines within a spectrum together with its continuum shape. Spectral lines that are typically anti-correlated will appear anti-correlated in the the second eigenspectrum (e.g., one with positive emission and the other as an absorption feature). Figure 20 plots the first three eigenspectra (with the first eigenspectrum the lower spectrum on the plot) with the typical emission and absorption lines identified by the SDSS spectroscopic pipelines (Stoughton et al. 2002) overlaid. The 2nd and 3rd eigenspectra are flipped and offset by an arbitrary amount to improve the clarity of this figure.

What is immediately apparent from this figure is that the majority of the nebula lines are highly correlated. An increase in the star formation rate within a galaxy will result in a general increase in the luminosity of all emission lines. While we expect this correlation in the hydrogen lines, it does not necessarily have to be the case for other lines such as [O III]: the physical processes that give rise to these lines are different (i.e., radiative vs collisional excitation). The most obvious anti-correlation arises for the Na D line at $5800 \AA$. The first eigen-component shows the sodium absorption (commonly associated with neutral gas at a temperature of a few thousand degrees) to be

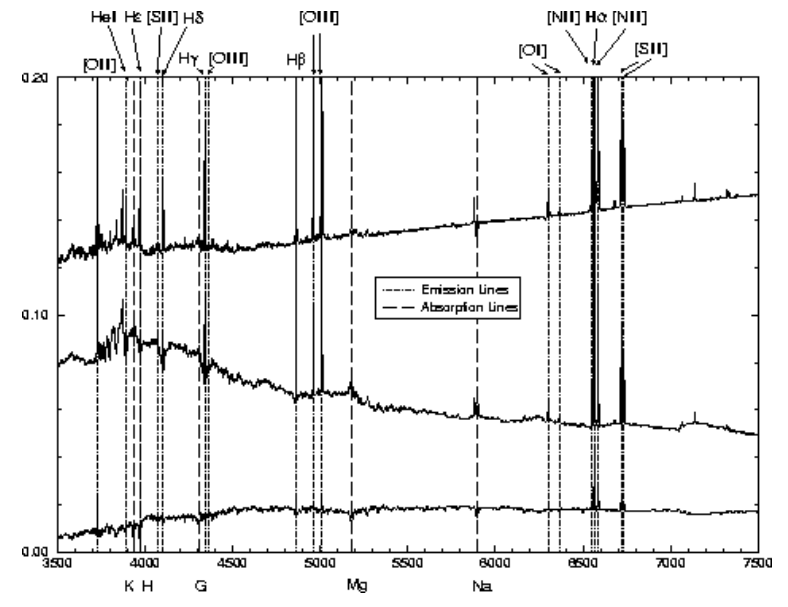

Fig. 20.- The 1st, 2nd and 3rd eigenspectra overlaid on the emission and absorption lines identified by the SDSS spectroscopic pipeline.

present in the mean spectrum of galaxies. From the second eigenspectrum, we see that as the star formation rate of the galaxy increases (i.e., we add the second component to the mean spectrum increasing the emission line strengths) the intensity of the Na D absorption line decreases. If the majority of the Na line comes from stellar lines (averaged over the 157,000 galaxies in this sample) then this relation is to be expected due to the increase in the population of $\mathrm{O}$ stars with increasing star formation rate. A similar relation is seen for the $\mathrm{Mg}$ triplet.

Considering the eigen-components individually we see that the mean galaxy spectrum for the main galaxy sample has a significant component in the $\mathrm{H} \alpha$ and $[\mathrm{N} \mathrm{II}]$ emission lines with weaker emission from [O III] and no real evidence for Balmer emission below $\mathrm{H} \beta$. Adding in the second eigenspectrum has the result of increasing the overall star formation within a galaxy (i.e., both the blue continuum increases together with the nebular emission lines). The second eigenspectrum has very strong Balmer absorption indicative of post starburst activity within a galaxy. The third component is dominated by line emission. There is very little of the stellar absorption of the Balmer emission lines as it is seen in the second eigenspectrum. A combination, therefore, of the first and third component will enable the reconstruction of pure absorption or emission line spectra. Within the third component the $\mathrm{Ca} \mathrm{K}$ and $\mathrm{Ca} \mathrm{H}$ absorption 
lines are strongly anti-correlated with the emission lines. Increasing the contribution of the third eigenspectrum has the net effect of increasing the line emission together with decreasing the strong absorption line features. This result is understood by the fact that absorption features in a galaxy are mainly due to older stellar populations, and many emission lines, especially nebular lines, are due to the ionization of the interstellar medium within the galaxy by hot stars.

It is, therefore, clear that the correlations present within the eigenspectra provide a reasonable description of the physical processes that occur within typical galaxy spectra. A more detailed description of these correlations will be the subject of a followup paper.

\subsection{Stellar-absorption of the Hydrogen Emission Lines}

Perhaps the most striking feature within these spectra is that the second eigenspectrum shows the hydrogen emission lines in $\mathrm{H} \epsilon, \mathrm{H} \delta, \mathrm{H} \gamma$, and $\mathrm{H} \beta$ exhibiting stellar absorption. The clarity of this effect comes from the high resolution of the SDSS spectroscopic data (relative to other large spectroscopic samples such as the $2 \mathrm{dF}$ ) together with the accurate control we have on the spectrophotometric calibration of the individual spectra. Figure 21 shows an enlarged region of interest for the first four eigenspectra. Comparatively, $\mathrm{H} \beta$ is the weakest in terms of this effect, while $\mathrm{H} \alpha$ shows no apparent effect. The absorption features are also observed in higher-order modes, but they are not shown here since the first few modes dominate. We find that the majority of the signal for the stellar absorption comes from the second eigen-component. There is a smaller contribution from the fourth component but the contribution from this component describes the variation in the widths of the hydrogen lines rather than their amplitudes.

As we have shown in Figure 11 using just three modes we can recover galaxy spectra with and without strong stellar absorption. The consequences of this are two-fold. The fourth eigencomponent appears not to contribute significantly to the stellar absorption signal, as noted above. Secondly, the fact that just two modes can recover the shape of the stellar absorption suggests that the mechanism that describes the magnitude

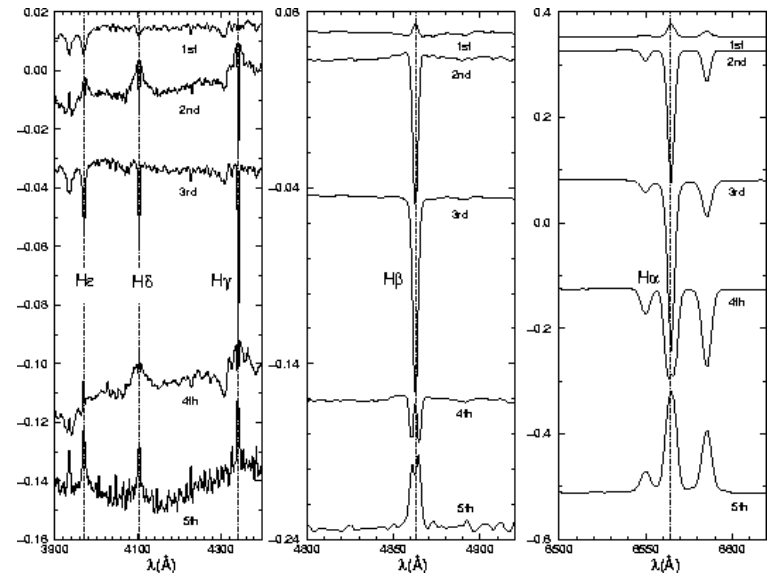

Fig. 21. - Stellar absorptions of hydrogen emission lines present in the eigenspectra. The eigenspectra are arbitrary shifted for clarity.

of this process is, on average, relatively simple (as would be expected given the correlation between the spectral properties and stellar composition of the galaxies). This would imply that modeling the stellar absorption and correcting for its effect on the emission line properties of galaxies should be a straightforward process in a statistical sense, even in the presence of low signal-to-noise data.

\section{The Effect of a Fixed Aperture}

The SDSS uses a fixed aperture of $3^{\prime \prime}$ diameter for its spectroscopic observations. This can, in principal, lead to biases in the current spectral classification scheme if, for example, a fiber samples only the central bulge of a nearby intermediate or late-type galaxy (resulting in the assignment of an early-type spectral class). As this effect depends on the apparent size of a galaxy when compared to the fiber diameter it has the potential to induce redshift and luminosity dependent biases in any analysis using the KL classifications (Kochanek et al. 2000). Studies of the effect of aperture bias on observed parameters (e.g., starformation rate) can be found in, e.g., Baldry et al. (2002), Pérez-González et al. (2003) and Brinchmann et al. (2003). The questions we address here are: $(i)$ is there an aperture bias using the KL approach $(i i)$ how can we quantify this bias and (iii) can we correct for the aperture effects to obtain bias-free galaxy types?

We estimate the effect of aperture bias by cal- 
culating, for a given galaxy, the difference in the classification (in this case the $\phi_{K L}$ angle) derived from the total galaxy flux compared to that derived from the central 3 arcseconds. The dependence of this classification error on the redshift and the physical size of the galaxy serves to quantify the bias in our sample. We assume that the apparent diameter of each galaxy can be approximated as twice the Petrosian half-light radius (petro50) in the r-band. The physical sizes of the galaxies are then calculated by assuming $\Omega_{m}=0.3$, $\Omega_{\Lambda}=0.7$ and $H_{0}=71$. The aperture magnitudes of all galaxies are initially k-corrected to redshift $z=0.1$ using the code by Blanton et al. (2003b) version 1.16 prior to estimating the spectral types. Type assignment for the total flux and fiber flux is performed using the photometric redshift code of Connolly et al. (1999b). The input spectral templates are constructed as linearcombinations of the first 3 eigenspectra from this work, with the resolution in both $\phi_{K L}$ and $\theta_{K L}$ set to $2^{\circ}$. In the following discussion we will express the distance dependence of the relation as function of $z / z_{\max }$, where $z_{\max }$ is the highest redshift at which a galaxy of a given absolute magnitude would pass the sample selection criteria. This provides a pseudo volume independent analysis.

Figure 22 shows the difference in the classifications of galaxies, $\phi_{K L}($ total $)-\phi_{K L}\left(3^{\prime \prime}\right)\left(\equiv D \phi_{K L}\right)$, as a function of $z / z_{\max }$ and galaxy type. The bin sizes of smoothing are 0.02 in $z / z_{\max }$ and $2^{\circ}$ in $D \phi_{K L}$. Galaxies of sizes from $0-100 \mathrm{kpc}$ are included, whereas galaxies of $\phi_{K L}($ total $)<-40^{\circ}$ are excluded for there are less then $1 \%$ of them. Lighter components in the greyscale image correspond to the fraction of galaxies that would be classified as an earlier type (i.e., redder) if the total flux was used rather than the 3 arcsec flux. Darker components correspond to galaxies that are of later type (bluer) when using the total flux. The percentages of galaxies residing within these contours are listed in Table 3. From our repeatability test, the mean signal-to-noise limited classification is $<\left|\delta\left(\phi_{K L}\right)\right|>=2.35$. With the assumption that the typical signal-to-noise limit in the $\phi_{K L}$ angle estimation for the whole galaxy is the same as that for the inner $3^{\prime \prime}$, the derived signalto-noise limit in $D \phi_{K L}$ is $2 \times<\left|\delta\left(\phi_{K L}\right)\right|>\approx 5$. There are about half of the galaxies $(\approx 40 \%)$ in our sample in which the type-differences are within the estimated signal-to-noise limit.

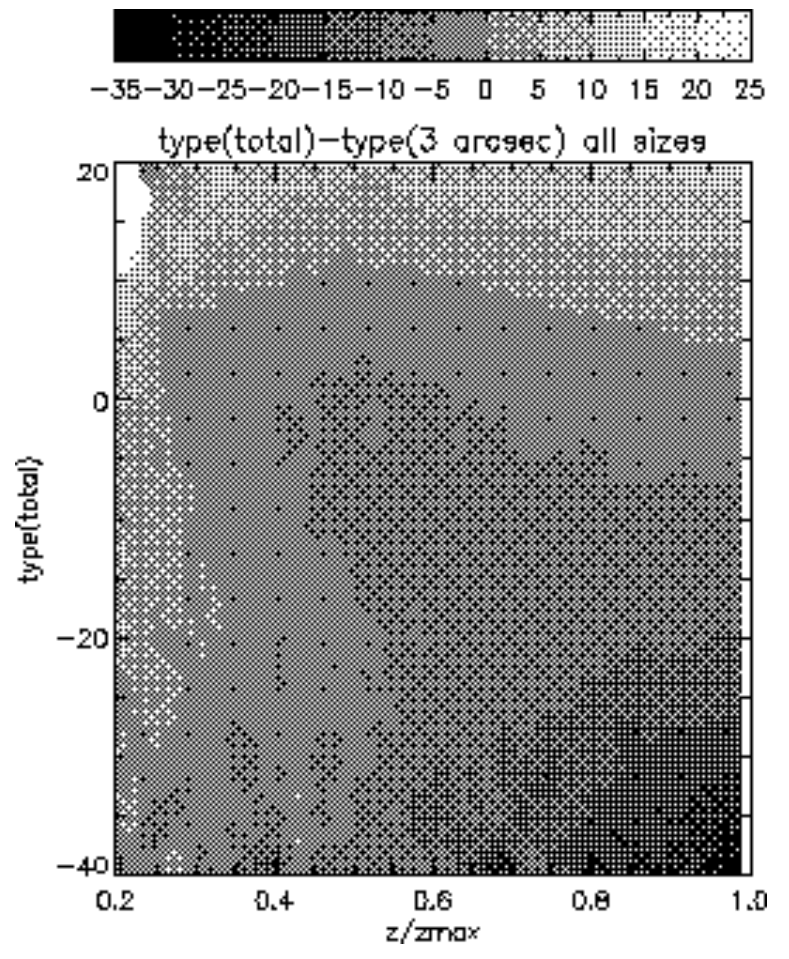

Fig. 22. - The greyscale contour of the difference in the classification $\left(D \phi_{K L}\right)$ for the total flux and for the inner $3^{\prime \prime}$ region as a function of $z / z_{\max }$. The ordinate is the type assigned from the total flux.

Aperture effects on the spectral classification clearly exist. For blue galaxies (i.e., $\phi_{K L} \approx$ $\left.0^{\circ}--40^{\circ}\right), D \phi_{K L}$ increases for nearby galaxies. This is to be expected as the flux from the inner 3 arcsec is more likely to be dominated by the presence of a bulge component. Similarly, for galaxies classified as red based on their total flux (i.e., $\phi_{K L} \approx 0^{\circ}-20^{\circ}$ ), errors in the classification angles $D \phi_{K L}$ increase rapidly with decreasing distance (i.e., $\left.z / z_{\max }<0.25\right)$. This implies that the cores of red galaxies are redder than the color estimated from the total flux.

The dependence of the aperture bias on the physical size of a galaxy is illustrated in Figure 23. We divide the above sample into 6 ranges of galaxy type, from the reddest in Figure (a) to the bluest in Figure (f). The differences in the classification angles $D \phi_{K L}$ are plotted in each figure as a function of $z / z_{\max }$ for physical sizes ranging from 
$0-100 \mathrm{kpc}$ (black line), $10-15 \mathrm{kpc}$ (dotted line) and $30-35 \mathrm{kpc}$ (dashed line). The two horizontal lines mark the uncertainty on the classification due to the survey signal-to-noise limits. For the red galaxies in Figure (a) to (c) the bias is constant or decreases with effective distance and is, essentially, negligible when compared to the uncertainties on the classification. For distances $z / z_{\max }<0.25$, the bias is above the signal-to-noise limit so that the type deduced from the total flux is redder than that from the central $3^{\prime \prime}$.

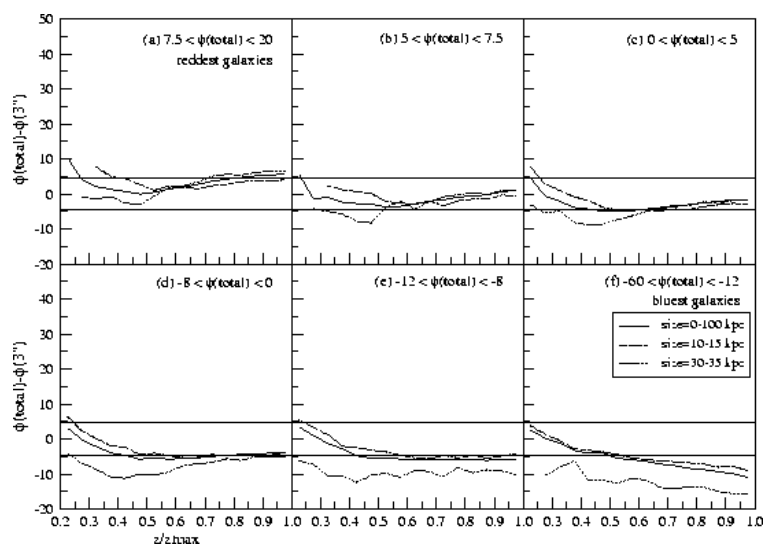

Fig. 23. - The difference in the classification for the whole galaxy and the inner $3^{\prime \prime}$ region as a function of $z / z_{\max }$ from the reddest (a) to the bluest (f) galaxies. In each sub-figure the galaxies are of sizes $0-100 \mathrm{kpc}$ (solid line), $10-15 \mathrm{kpc}$ (dotted line) and $30-35 \mathrm{kpc}$ (dashed line).

As we would expect Figures (b) and (c) show a dependence on galaxy size for the classifications with larger galaxies exhibiting a redder classification when only considering the $3 \operatorname{arcsec}$ flux. This size and redshift dependence extends to the blue galaxies (Figure (d) through (f)). For these galaxies, however, a more pronounced dependency is shown on the classification bias with galaxy size. Overall there is a general aperture bias for all physical sizes of galaxy $(0-100 \mathrm{kpc})$ that approaches the intrinsic error on the classification as the effective redshift $z / z_{\max }$ approaches unity. The exception to this arises when we consider galaxies with prominent emission lines (Figure (f)) which, counter-intuitively, exhibit larger bias the more distant they are. One of the possible reasons for this is our use of three eigenspectra in constructing the spectral templates, whereas 10 modes and more are typically required to accurately reconstruct these observed spectra.

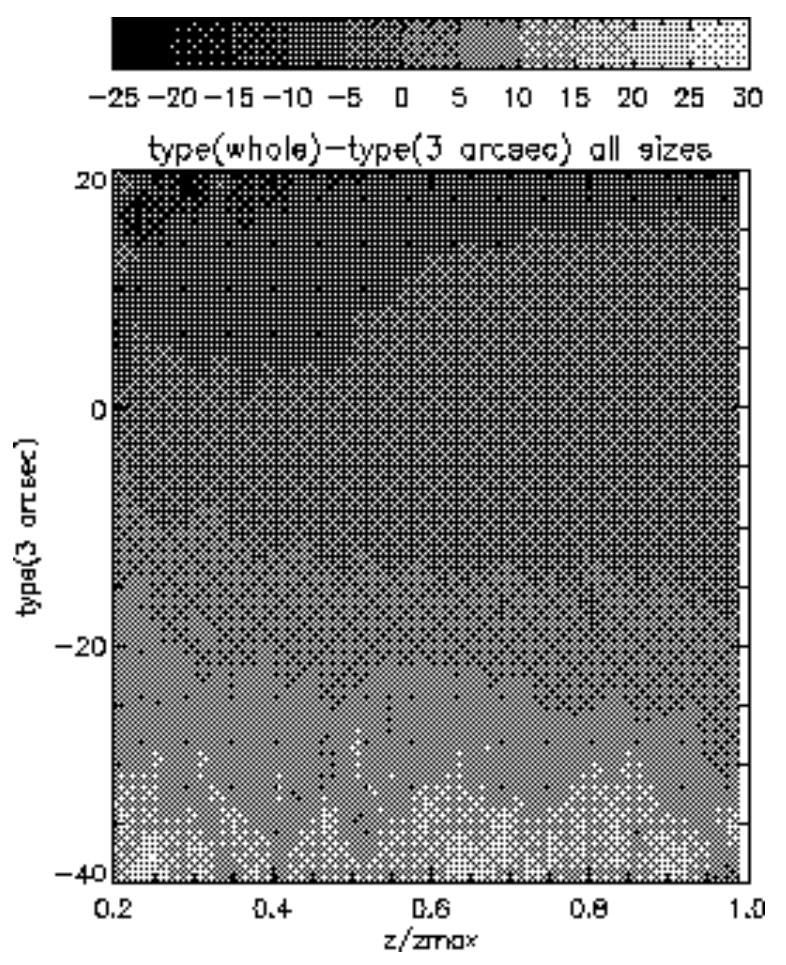

Fig. 24. - Same as Figure 22 except that the ordinate is the type for the inner $3^{\prime \prime}$ of each galaxy. This serves as the look-up table with which the aperture bias can be corrected.

While we observe an aperture bias in the SDSS sample it is relatively small when compared to the intrinsic classification errors and is essentially negligible for most galaxies. Moreover its dependence on size and redshift is relatively mild and straightforward to correct. For those galaxies with nonnegligible bias a simple correction can be made using the lookup table shown in Figure 24, which is identical to Figure 22 with the exception that the ordinate axes is the spectral type inside $3^{\prime \prime}$, $\phi_{K L}\left(3^{\prime \prime}\right)$. Given the $\phi_{K L}\left(3^{\prime \prime}\right)$ and $z / z_{\max }$ the correction to our spectral classification can be determined directly from Figure 24 with the sizeaveraged bias.

\section{Conclusions and Outlook}

From the application of Karhunen-Loève transform, an objective classification of $\approx 170,000$ galaxy spectra in the SDSS is performed. With 
a quantitative convergence criteria defined, gappy galaxy spectra can be repaired and KL eigenspectra and eigencoefficients derived. For most of the galaxy types, three eigenspectra are sufficient for describing the continua and emission lines to a high degree of accuracy with a maximum error in line-reconstructions of approximately 10\%. Typically ten modes are needed in the reconstruction of galaxies with extreme emission lines with errors of $15-25 \%$ in the line fluxes. We find that a two-parameter $\left(\phi_{K L}, \theta_{K L}\right)$-classification scheme can discriminate between spectra corresponding to all spectral types used in the current classification scheme (including galaxies with extreme emission lines). This classification is robust to repeat observations (at a level of a few degrees in the classification angles) due to the accurate spectrophotometric calibration of the SDSS data set. We find a weak dependence in the classification on the signal-to-noise of the spectra. This effect is, however, smaller than the typical dispersion between repeat observations and is negligible at signal-to-noise levels at which the SDSS spectra are defined as being of survey quality.

We find that there exists a minimum number of randomly selected spectra that are necessary to statistically represent the information within the full sample (i.e., to be representative of the true distribution of galaxies). For a set of ten eigenspectra (i.e., ten eigenspectra enable the reproduction of both quiescent and active galaxies) the number of spectra required is around 3000 to 4000. This is due to the need to sample a minimum number of randomly selected galaxies in order to include galaxies with extreme emission line properties in our data set (as they comprise only $0.1 \%$ of the full galaxy sample).

We find that the bias on the spectral classification due to the fixed aperture spectroscopy is, on average, small and is negligible for all galaxies except for the reddest galaxies that are very close by $\left(z / z_{\max }<0.3\right)$ and for those galaxies that are large physically $(>30 \mathrm{kpc})$ with prominent emission lines. A look-up table is constructed for the correction of this bias.

There are several future directions related to this work. With the present continuous classification scheme, which simplifies the distribution of galaxies into a handful of parameters, studies of the statistics of the physical properties of galaxies become more tractable. The clustering and spectral properties of these classifications will be addressed in a future paper. The generality of these techniques are applicable to any set of spectra and has been recently applied to the SDSS QSO cata$\log$ (Yip et al. 2003, 2004).

We thank Mariangela Bernardi, Ravi Sheth and Michael Blanton for comments and discussions. CWY is partially supported by Zaccheus Daniel Fellowship. AJC and CWY acknowledge partial support from an NSF CAREER award AST99 84924, a NASA LTSA grant NAG5 8546 and a NSF ITR award 0121671.

Funding for the creation and distribution of the SDSS Archive has been provided by the Alfred P. Sloan Foundation, the Participating Institutions, the National Aeronautics and Space Administration, the National Science Foundation, the U.S. Department of Energy, the Japanese Monbukagakusho, and the Max Planck Society. The SDSS Web site is http://www.sdss.org/.

The SDSS is managed by the Astrophysical Research Consortium (ARC) for the Participating Institutions. The Participating Institutions are The University of Chicago, Fermilab, the Institute for Advanced Study, the Japan Participation Group, The Johns Hopkins University, Los Alamos National Laboratory, the Max-Planck-Institute for Astronomy (MPIA), the Max-Planck-Institute for Astrophysics (MPA), New Mexico State University, University of Pittsburgh, Princeton University, the United States Naval Observatory, and the University of Washington. 


\section{REFERENCES}

Bailer-Jones C. A. L., Irwin M. \& von Hippel T. 1998, MNRAS, 298, 361.

Baldry I. K.,Glazebrook K. B., Carlton M. et al. 2002, ApJ, 536, 68.

Blanton M. R., Lupton R. H., Maley F. M. et al. 2002, submitted to AJ.

Blanton M. R., Hogg D. W., Bahcall N. A. et al. 2003a, ApJ, 594, 186.

Blanton M. R., Brinkmann J., Csabai I. et al. 2003b, AJ, 125, 2348.

Boroson T. A. \& Green R. F. 1992, ApJ Supp. Series, 80, 109.

Brinchmann J., Charlot S., White S. D. M. et al. 2003, submitted to MNRAS.

Bromley B. C., Press W. H., Lin H. \& Kirshner R. 1998, ApJ, 505, 25.

Castander F. J., Nichol R. C., Merrelli A. 2001, AJ, 121, 2331.

Colless M. M., Dalton G., Maddox S. et al. 2001, MNRAS, 328, 1039.

Connolly A. J., Genovese C., Moore A. W. et al. 2000, submitted to AJ.

Connolly A. J., Szalay A. S., Bershady M. A., Kinney A. L. \& Calzetti D. 1995, ApJ, 110, 1071.

Connolly A. J. \& Szalay A. S. 1999a, ApJ, 117, 2052.

Connolly A. J., Budavári T., Szalay A. S., Csabai I. \& Brunner R. J. 1999b, in ASP Conf. Ser. 191, Photometric Redshifts and High Redshift Galaxies, ed. Weymann R. J., StorrieLombardi L. J., Sawicki M., \& Brunner R. J. (San Francisco: ASP), 13.

Efstathiou G. \& Fall M. S. 1984, MNRAS, 206, 453.

Eisenstein D. J., Annis J., Gunn J. E. et al. 2001, AJ, 122, 2267.

Eisenstein D. J. Hogg D. W., Fukugita M. et al. 2003, ApJ, 585, 694.
Everson R. \& Sirovich L. 1994, J.Opt.Soc.Am.A, 12, No.8, 1657.

Folkes S. R., Lahav O. \& Maddox S. J. 1996, MNRAS, 283, 651.

Folkes S. R., Ronen S., Price I. et al. 1999, MNRAS, 308, 459.

Francis P. J., Hewett P. C., Foltz C. B. \& Chaffee F. H 1992, ApJ, 398, 476.

Fukugita M., Ichikawa T., Gunn J. E. et al. 1996, AJ, 111, 1748.

Galaz G. \& de Lapparent V. 1998, A\&A, 332, 459.

Gunn J. E., Carr M., Rockosi C. et al. 1998, AJ, 116, 3040.

Hogg D. W., Finkbeiner D. P., Schlegel D. J. \& Gunn J. E. 2001, AJ, 122, 2129.

Hubble E. 1926, ApJ, 64, 321.

Kennicutt R. C., Jr. 1992, ApJ Supp. Series, 79, 255.

Kochanek C. S., Pahre M. A. \& Falco E. E. astro$\mathrm{ph} / 0011458$.

Lupton R., Gunn J. E., Ivezić Z., Knapp G. R. \& Kent S. 2001, in ASP Conf. Ser. 238, Astronomical Data Analysis Software and Systems X, ed. Harnden F. R. Jr., Primini F. A., \& Payne H. E. (San Francisco: Astr. Spc. Pac.), p. 269.

Merzbacher E. 1970, Quantum Mechanics, second edition, John Wiley\&Sons.

Murtagh F. \& Heck A. 1987, Multivariate data analysis, Astrophysics and Space Science Library No 131, Dordrecht: Reidel.

Pérez-González P. G., Zamorano J., Gallego J., Aragón-Salamanca A. \& Gil de Paz A. 2003, ApJ, 591, 827.

Pier J. R., Munn J. A., Hindsley R. B. et al. 2002, AJ, 125, 1559.

Richards G. T., Fan X., Newberg H.J. et al. 2002, AJ, 123, 2945.

Ronen S., Aragón-Salamanca A. \& Lahav O. 1999, MNRAS, 303, 284. 
Shimasaku K., Fukugita M., Doi M. et al. 2001, AJ, 122, 1238.

Singh H. P., Gulati R. K. \& Gupta R. 1998, MNRAS, 295, 312.

Smith J. A., Tucker D. L., Kent S. et al. 2002, AJ, 123, 2121.

Sodré L. Jr \& Cuevas H. 1997, MNRAS, 287, 137.

Stoughton C., Lupton R. H., Bernardi M. et al. 2002, ApJ, 123, 485.

Strauss M. A., Weinberg D. H., Lupton R. H. et al. 2002, AJ, 124, 1810.

Strateva I., Ivezic Z., Knapp G. R. et al. 2001, ApJ, 122, 1861.

Yip. C. W., Connolly A. J., Vanden Berk D. E. et al. 2003, in ASP Conf. Ser. 311, AGN Physics with the Sloan Digital Sky Survey, ed. Hall P. B. \& Richards G. T..

Yip. C. W., Connolly A. J., Vanden Berk D. E. et al. 2004, in prep (SDSS Publication No. $331)$.

York D. G., Adelman J., Anderson J. E. Jr. et al. 2000, ApJ, 120, 1579.

This 2-column preprint was prepared with the AAS IATEX macros v5.0. 


\begin{tabular}{|c||c|c|}
\hline$\left(\phi_{K L}^{s}, \phi_{K L}^{e}, \theta_{K L}^{s}, \theta_{K L}^{e}\right)$ & Galaxy Type & Number [Number ratio relative to Sa] \\
\hline \hline$(7.5,20,86,92)$ & $\mathrm{E} / \mathrm{S} 0$ & $6599[0.34]$ \\
\hline$(5,6,80,100)$ & $\mathrm{Sa}$ & $19543[1.00]$ \\
\hline$(0,2,80,100)$ & $\mathrm{Sb}$ & $13872[0.71]$ \\
\hline$(-12,-8,80,100)$ & $\mathrm{Sbc} / \mathrm{Sc}$ & $11979[0.61]$ \\
\hline$(-40,-30,80,100)$ & $\mathrm{Sm} / \mathrm{Im}$ & $140[0.0072]$ \\
\hline$(-60,-40,120,135)$ & $\mathrm{SBm}$ & $135[0.0069]$ \\
\hline \hline
\end{tabular}

Table 1: The number of galaxies in the range $\left(\phi_{K L}^{s}, \phi_{K L}^{e}, \theta_{K L}^{s}, \theta_{K L}^{e}\right)$. These data are a subset of the full sample. The galaxy types listed are the possible morphological types, estimated by comparing the spectral features of the mean spectrum constructed in each range with spectra in (Kennicutt 1992) and therefore they are for reference only. 


\begin{tabular}{|r|c|}
\hline$m$ (number of modes) & weight (normalized by total weight) \\
\hline \hline 1 & 0.9594 \\
2 & 0.9784 \\
3 & 0.9815 \\
5 & 0.9837 \\
8 & 0.9849 \\
10 & 0.9852 \\
20 & 0.9855 \\
50 & 0.9860 \\
100 & 0.9867 \\
500 & 0.9908 \\
1000 & 0.9940 \\
\hline
\end{tabular}

Table 2: The relative weights of the eigenspectra. The first 3 eigenspectra comprise about $98 \%$ of the total sample variance.

\begin{tabular}{|c|c|}
\hline $\begin{array}{c}D \phi_{K L} \text {-range } \\
\text { (degree) }\end{array}$ & $\begin{array}{c}\text { fraction of galaxies } \\
(0.1 \% \text { accuracy })\end{array}$ \\
\hline \hline$-20<D \phi_{K L}<=-10$ & $18.5 \%$ \\
$-10<D \phi_{K L}<=-5$ & $16.4 \%$ \\
$-5<D \phi_{K L}<=-2.5$ & $9.2 \%$ \\
$-2.5<D \phi_{K L}<=0$ & $17.7 \%$ \\
$0<D \phi_{K L}<=2.5$ & $7.3 \%$ \\
$2.5<D \phi_{K L}<=5$ & $6.1 \%$ \\
$5<D \phi_{K L}<=10$ & $12.1 \%$ \\
$10<D \phi_{K L}<=20$ & $7.2 \%$ \\
\hline
\end{tabular}

Table 3: The number of galaxy spectra in our sample with the specified values of aperture bias $D \phi_{K L}$. 